\title{
RNA-seq analysis of the influence of anaerobiosis and FNR on Shigella flexneri
}

\author{
Marta Vergara-Irigaray ${ }^{1,2}$, Maria C Fookes ${ }^{3}$, Nicholas R Thomson ${ }^{3}$ and Christoph M Tang 1,2* $^{\text {. }}$
}

\begin{abstract}
Background: Shigella flexneri is an important human pathogen that has to adapt to the anaerobic environment in the gastrointestinal tract to cause dysentery. To define the influence of anaerobiosis on the virulence of Shigella, we performed deep RNA sequencing to identify transcriptomic differences that are induced by anaerobiosis and modulated by the anaerobic Fumarate and Nitrate Reduction regulator, FNR.

Results: We found that 528 chromosomal genes were differentially expressed in response to anaerobic conditions; of these, 228 genes were also influenced by FNR. Genes that were up-regulated in anaerobic conditions are involved in carbon transport and metabolism (e.g. ptsG, manX, murQ, cysP, cra), DNA topology and regulation (e.g. ygiP, stpA, hns), host interactions (e.g. yciD, nmpC, slyB, gapA, shf, msbB) and survival within the gastrointestinal tract (e.g. shiA, ospl, adiY, cysP). Interestingly, there was a marked effect of available oxygen on genes involved in Type III secretion system (T3SS), which is required for host cell invasion and pathogenesis. These genes, located on the large Shigella virulence plasmid, were down regulated in anaerobiosis in an FNR-dependent manner. We also confirmed anaerobic induction of csrB and csrC small RNAs in an FNR-independent manner.
\end{abstract}

Conclusions: Anaerobiosis promotes survival and adaption strategies of Shigella, while modulating virulence plasmid genes involved in T3SS-mediated host cell invasion. The influence of FNR on this process is more extensive than previously appreciated, although aside from the virulence plasmid, this transcriptional regulator does not govern expression of genes on other horizontally acquired sequences on the chromosome such as pathogenicity islands.

\section{Background}

Shigella flexneri is a Gram-negative bacterium that causes dysentery, an acute human rectocolitis that usually results in destruction of the intestinal mucosa and bloody diarrhoea. The ability of this pathogen to invade epithelial cells at the colonic and rectal mucosal surface is a key determinant in the establishment of the disease. This is mediated by a Type III secretion system (T3SS) encoded on the large Shigella virulence plasmid [1,2]. The T3SS acts like a molecular syringe that delivers molecules directly from the bacterial cytoplasm into host cells via a needlelike structure $[1,2]$. However, before the bacterium reaches the large intestine and invades mucosal epithelial cells, Shigella must successfully survive the hostile conditions found in the gastrointestinal tract. Therefore the capacity

\footnotetext{
* Correspondence: christoph.tang@path.ox.ac.uk

'Sir William Dunn School of Pathology, Oxford University, Oxford, United Kingdom

${ }^{2}$ Centre for Molecular Microbiology and Infection, Imperial College London, London, United Kingdom

Full list of author information is available at the end of the article
}

of the bacterium to adapt to anaerobiosis, changes in $\mathrm{pH}$, resist antimicrobial peptides, and acquire nutrients is essential for its pathogenesis $[3,4]$.

Anaerobiosis is known to influence the virulence of several enteric pathogens including Shigella, Escherichia coli, Salmonella spp., Vibrio cholerae and Yersinia enterocolitica [5-13]. In particular, S. flexneri has been shown to be primed for invasion in anaerobic conditions, in which it expresses longer T3SS needles while reducing Ipa (invasion plasmid antigen) effector secretion; this results from FNR-mediated repression of the virulence plasmid genes, spa32 and spa33 [7]. FNR is a major regulator of anaerobic metabolism that is inactivated by the presence of oxygen. Its function depends on the integrity of its $\mathrm{O}_{2}$-sensitive [4Fe-4S] cluster, which is required for FNR dimerization and thence site-specific DNA binding and transcriptional regulation [14]. One RNA deep sequencing (RNA-seq) and several microarray studies have been performed to characterise the extent of the FNR regulon in E. coli and other Gram negative 
pathogens such as Salmonella enterica and Neisseria gonorrhoeae [15-20]. In E. coli, there were significant discrepancies between studies even when the same strain was examined. However some differences could be attributed to the use of media containing high levels of glucose, which represses expression from some FNRactivated promoters, and the delayed growth rate of mutants lacking FNR compared with wild-type strains under anaerobic conditions [16].

Here we define the regulatory role of oxygen and FNR in S. flexneri. We have applied two powerful wholetranscriptome approaches, RNA-seq complemented with Flow cell Reverse Transcription sequencing (FRT-seq), in which there is no amplification during library preparation, to quantify differences in gene expression induced by anaerobiosis and to define the contribution of FNR in this process. We found that Shigella grown anaerobically exhibits global transcriptional changes compared to when grown aerobically, with marked changes in metabolic and transport genes, as well as those involved in regulatory and virulence functions. Importantly, transcription from the Shigella virulence plasmid is extensively modified in anaerobiosis, with most of T3SS-related genes being down regulated in the absence of oxygen in an FNR-dependent manner, demonstrating that this highly conserved regulator of metabolism also controls the horizontally-acquired virulence genes on the plasmid, but not on the chromosome, in this important human pathogen.

\section{Results}

\section{Growth conditions and RNA sequencing strategies}

To determine the response of Shigella to anaerobiosis and the role of FNR in this process, we employed RNAseq to compare the transcriptional profiles of wild type S. flexneri M90T and its $\Delta f n r$ mutant grown in LuriaBertani (LB) medium in the presence and absence of oxygen. Constantinidou et al. designed a supplemented, minimal salts medium (including LB) in which an E. coli fnr mutant exhibited similar growth as the parental strain in the absence of oxygen [16]. However, this medium did not support the growth of S. flexneri M90T. On the other hand, enriched-glucose media have been shown to repress some FNR-activated promoters [16]. Therefore, we chose LB with no added glucose for our experiments. Particular attention was paid to ensure that the culture volume, agitation, temperature and the growth stage of bacteria did not differ in aerobic and anaerobic conditions. Cultures were grown to an Optical Density at $600 \mathrm{~nm}\left(\mathrm{OD}_{600}\right)$ of 0.2 to avoid a reduction in the concentration of dissolved oxygen tension and total depletion of sugars that occurs during exponential growth $[21,22]$. Furthermore until reach $\mathrm{OD}_{600}$ of 0.2 under anaerobiosis, there was no obvious delay in growth rate of the $\Delta f n r$ mutant in relation to the wild-type strain (See Additional file 1: Figure S1). Three biological replicates were performed per strain in each condition, and differential expression between conditions was analysed with the DESeq R statistical package.

To assess the reproducibility of results obtained with RNA-seq data and to further characterise the role of FNR, the Shigella FNR regulon under anaerobiosis was also examined using FRT-seq, an alternative sequencing approach in which cDNA synthesis is performed on the sequencing flowcell thereby avoiding the possible PCR biases generated during library preparation using standard RNA-seq methods [23]. FRT-seq confirmed $77 \%$ of the genes found differentially expressed by RNA-seq, showing a robust concordance between the two techniques. Due to its higher sensitivity, FRT-seq detected more genes whose transcription was significantly influenced by the absence of FNR than RNA-seq (See Additional file 1: Table S2). A complete catalogue of significant differences is shown in Additional material (See Additional file 1: Tables S1 and S2) as well as a summary of the mapping statistics (See Additional file 1: Table S3). To confirm the results obtained by global analysis of the transcriptional profile, we performed strand-specific qRT-PCR to analyse mRNA levels of several genes found to be differentially expressed under anaerobic and aerobic growth conditions.

\section{Identification of novel chromosomal genes influenced by the absence of oxygen in S. flexneri}

Analysis of the RNA-seq data revealed that 528 chromosomal genes were differentially expressed by wild-type S. flexneri M90T grown under anaerobic conditions compared with aerobic conditions, with 363 genes being up-regulated, and 165 genes down-regulated. Additional file 1: Table S1 shows these genes classified into functional categories based on the database of Clusters of Orthologous Groups (COGs) [24]. As expected, most of the genes differentially expressed were related to energy production and metabolism (53\%). The remaining genes were involved in cellular processes and signalling (15\%), information storage and processing $(8 \%)$ or were poorly characterized (24\%). RNA-seq data also showed that from the above 528 differentially expressed genes, 228 genes (43\%) were influenced by the absence of FNR under anaerobic conditions (See Additional file 1: Table S1).

Importantly the majority of genes that we found to be anaerobically induced/repressed have been identified in previous microarray studies with other enteric pathogens examining the effect of oxygen on the transcriptome and/or the two main anaerobic regulators, FNR and ArcA [6,16-20,25]. Consistent with previous work, we found increased expression of genes involved in anoxic carbon metabolism (focA-pfl, yfiD, fdnG, gldA, aspA, fumB, ans $B)$, respiratory pathways (glpABC, nap, nir, 
$c c m, n r f A B C, f r d)$, production of hydrogenases (hyb, hya, hyc, hyp), fermentation ( $a d h E$, ackA-pta, fdhF) and acid response (adiA, adiY, yjdE, $\operatorname{gad} A$, hdeAB) under anaerobiosis (Additional file 1: Table S1) [6,16-18,20,25,26]. Our analysis also identified several anaerobically repressed genes that have been previously characterised [6,16-20,25]. These genes encode enzymes of the tricarboxylic acid cycle (ace, gltA, acn, icdA, $s d h$ ), aerobic dehydrogenases $(g l p D$, betBA, gcd, ald $A)$, transhydrogenases $(u d h A)$ and iron acquisition systems (exb, iuc, iutA, sit, suf, fep, fhu), and others (Additional file 1: Table S1) $[6,16-18,20,25,27,28]$.

The sensitivity of the direct sequencing approaches, RNA-seq and FRT-seq, compared with array-based methods enabled us to extend the repertoire of Shigella genes modulated by ambient oxygen. Table 1 shows all genes influenced by the presence of oxygen and not detected in previous microarray studies on $E$. coli and $S$. flexneri $[6,16-18,20,25]$. The effect of FNR mutation on the transcription of previous genes under anaerobiosis (assessed by RNA-seq and FRT-seq) is also shown in Table 1. Several members of the phosphoenolpyruvatecarbohydrate phosphotransferase system (PTS), involved in the transport and phosphorylation of sugars, were upregulated under anaerobic conditions. Examples include ptsHI, which encode the general PTS components phosphohistidine carrier protein (HPr) and Enzyme I (EI) respectively, and sugar-specific PTS components like ptsG and $\operatorname{man} X Y Z$ (involved in glucose transport), tre $B C$ (trehalose transport and hydrolysis), $m$ tlA (mannitol) and murQP that contribute to the uptake and catabolism of $N$-acetylmuramic acid [29-32]. Of note, the murQP operon, which is also involved in peptidoglycan recycling, showed an FNR-dependent expression pattern (Table 1, Figure 1A) [31].

The expression of other genes involved in transport displayed altered expression in anaerobiosis. For instance, emrD, coding for a drug transporter, cys $P$, involved in the binding and uptake of sulfate and thiosulfate, $y j c E$, coding for a $\mathrm{Na}^{+} / \mathrm{H}^{+}$exchanger, $y b g H$, which encodes a peptide transporter and genes involved in nucleoside transport and catabolism (tsx, nupC, nup $G$ and $u d p)$ are induced in anaerobiosis (Table 1, Figure 1A) [33-40].

We found several metabolic genes induced under anaerobic growth such as cra, coding for the catabolite repressor/activator protein, Cra, tpiA, encoding a key enzyme of the gluconeogenic and glycolytic pathways, gapA, involved in glycolysis, yehU/yehT, coding for a two component system involved in responses to carbon starvation, malT, the transcriptional activator of the genes responsible for uptake and metabolism of maltodextrins and $\operatorname{pro} A$, which encodes an enzyme in proline biosynthesis [41-47]. The expression of these genes was not FNR-dependent (Table 1, Figure 1A).
In addition to metabolism, we observed anaerobic upregulation of: genes involved in stress response such as $\operatorname{csp} C$; genes coding for outer membrane proteins (OMPs) such as NmpC, OmpA and SlyB; genes with global regulatory functions such as $y j g D$ that codes for RraB, which interacts with the endonuclease RNase E; $y f i A$, encoding a ribosome-associated protein that inhibits protein translation; and yejK, hns and its paralogue $\operatorname{stp} A$ coding for nucleoid-associated proteins responsible for chromosomal DNA compaction and global gene regulation [48-56]. Interestingly, anaerobic induction of $\operatorname{csp} C, n m p C$, slyB, yjgD, hns and $\operatorname{stp} A$ was dependent, at least in part, on FNR (Table 1, Figure 1B). Anaerobiosis can also down-regulate transcription. This is the case for fruBKA, encoding the fructose PTS [29] (Table 1, Figure 2).

The analysis of genes known to be influenced by anaerobiosis revealed further functions of FNR. This is the case for $y g i P$, encoding a nucleoid-associated protein induced under anaerobic growth conditions, which we found is FNR-dependent [57]. Furthermore, we observed that menDBCE, genes required for the biosynthesis of quinones with essential roles in anaerobic electron transport systems, are affected by the presence of FNR in contrast to E. coli (Table 1, Figure 1A and B) [58-60].

Our study revealed extended regulatory roles for FNR, such as in the biosynthesis of L-cysteine. Previous work has demonstrated that cysK, which encodes an enzyme in L-cysteine biosynthesis, is subject to FNR regulation and identified an FNR-like domain in cysJ, which encodes a component of the sulfite reductase [16,61]. Here, we found that loss of FNR affects the entire L-cysteine biosynthetic pathway including genes involved in the uptake and transport of sulfate (i.e. cysPUWAM), sulfate activation (cysDN), reduction to sulfide (cysJIH) and transformation into L-cysteine (cysK) (Table 1, Figure 1A, see Additional file 1: Table S2) [62-64].

\section{Reprogramming of T3SS related genes under anaerobic conditions}

Analysis of genes involved in Shigella virulence revealed that multiple genes on the Shigella virulence plasmid, including ipa-mxi-spa genes, were repressed under anaerobic growth in an FNR-dependent manner (Table 2). In contrast, only seven genes on the plasmid (yigB, ospI, shf, rfbU, virK, $m s b B$ and parA) were upregulated in the absence of oxygen; all of these are regulated by FNR except parA and yigB (Table 2). Figure 3 shows effect of oxygen on expression of genes on the virulence plasmid genes. These findings were confirmed by strand specific qRT-PCR for several genes (Figures 4 and 5). Since excess ParA levels compared with ParB can affect plasmid partitioning, we 
Table 1 Chromosomal genes differentially expressed in response to anaerobic conditions not previously published in E. coli and S. flexneri microarray analysis

\begin{tabular}{|c|c|c|c|c|c|}
\hline ORF ID ${ }^{a b}$ & Gene & Description & $\begin{array}{c}\text { RNA-seq } \\
\text { log2FC }\end{array}$ & $\begin{array}{c}\text { RNA-seq }^{c} \\
\log 2 \mathrm{FC}\end{array}$ & $\begin{array}{c}\text { FRT-seq }^{c} \\
\log 2 F C\end{array}$ \\
\hline & & & WT no $\mathrm{O}_{2} / \mathrm{O}_{2}$ & $\Delta$ fnr/WT no $\mathrm{O}_{2}$ & $\Delta f n r /$ WT no $\mathrm{O}_{2}$ \\
\hline
\end{tabular}

\section{Metabolism}

Energy production and conversion

\begin{tabular}{|c|c|c|}
\hline SF5M90T_1519 & & putative oxidoreductase, major subunit \\
\hline SF5M90T_3856 & yiaY & putative oxidoreductase \\
\hline SF5M90T_1560 & & putative oxidoreductase, major subunit \\
\hline SF5M90T_3333 & $p c k A$ & phosphoenolpyruvate carboxykinase \\
\hline SF5M90T_3877 & yiak & putative dehydrogenase \\
\hline SF5M90T_3374 & ugpQ & glycerophosphodiester phosphodiesterase, cytosolic \\
\hline SF5M90T_2534 & hmpA & dihydropteridine reductase, ferrisiderophore reductase activity \\
\hline SF5M90T_33 & caiB & I-carnitine dehydratase \\
\hline SF5M90T_3679 & $\operatorname{atpF}$ & membrane-bound ATP synthase, F0 sector, subunit b \\
\hline SF5M90T_3680 & $\operatorname{atp} E$ & membrane-bound ATP synthase, F0 sector, subunit c \\
\hline SF5M90T_3937 & $p p c$ & phosphoenolpyruvate carboxylase \\
\hline SF5M90T_579 & galt & galactose-1-phosphate uridylyltransferase \\
\hline SF5M90T_1419 & $y d j A$ & predicted oxidoreductase \\
\hline SF5M90T_2771 & ygaF & hydroxyglutarate oxidase \\
\hline SF5M90T_4044 & $g / t P$ & glutamate-aspartate symport protein \\
\hline SF5M90T_1603 & $r n f B$ & electron transport complex protein \\
\hline SF5M90T_2869 & $f l d B$ & flavodoxin 2 \\
\hline SF5M90T_1602 & $r n f A$ & $\mathrm{Na}+-$ translocating $\mathrm{NADH}$-quinone reductase subunit $\mathrm{E}$ \\
\hline SF5M90T_1011 & rutA & pyrimidine monooxygenase \\
\hline
\end{tabular}

Carbohydrate transport and metabolism

$\begin{array}{lcl}\text { SF4250 } & \text { treB } & \text { PTS system trehalose(maltose)-specific transporter subunits IIBC } \\ \text { SF5M90T_4160 } & \text { treC } & \text { trehalase 6-P hydrolase } \\ \text { SF5M90T_1379 } & \text { manX } & \text { PTS enzyme IIAB, mannose-specific } \\ \text { SF5M90T_1378 } & \text { manY } & \text { PTS enzyme IIC, mannose-specific } \\ \text { SF5M90T_1377 } & \text { manZ } & \text { PTS enzyme IID, mannose-specific } \\ \text { SF5M90T_3670 } & \text { rbsD } & \text { high affinity ribose transport protein } \\ \text { SF5M90T_1101 } & \text { ptsG } & \text { PTS system, glucose-specific IIBC component } \\ \text { SF5M90T_3491 } & \text { treF } & \text { cytoplasmic trehalase } \\ \text { SF5M90T_2419 } & \text { murP } & \text { PTS system N-acetylmuramic acid transporter subunits EIIBC } \\ \text { SF5M90T_3499 } & \text { pfkA } & \text { 6-phosphofructokinase I } \\ \text { SF5M90T_2096 } & & \text { fructose-bisphosphate aldolase } \\ \text { SF5M90T_1001 } & \text { agp } & \text { periplasmic glucose-1-phosphatase } \\ \text { SF5M90T_2887 } & \text { rpiA } & \text { ribosephosphate isomerase, constitutive } \\ \text { SF5M90T_2898 } & \text { pgk } & \text { phosphoglycerate kinase } \\ \text { SF5M90T_1403 } & \text { gapA } & \text { glyceraldehyde-3-phosphate dehydrogenase A } \\ \text { SF5M90T_2097 } & \text { yegT } & \text { putative nucleoside permease protein } \\ \text { SF5M90T_2897 } & \text { fba } & \text { fructose-bisphosphate aldolase, class II } \\ \text { SF5M90T_2404 } & \text { ptsH } & \text { PTS system protein HPr } \\ \text { SF5M90T_3850 } & \text { mtIA } & \text { PTS system, mannitol-specific enzyme IIABC components } \\ \text { SF5M90T_1640 } & \text { ydhC } & \text { putative transport protein }\end{array}$

3.80

3.18

3.06

1.99

1.93

1.89

1.47

1.32

1.04

1.04

0.91

0.77

$-1.17$

$-1.31$

$-1.32$

$-1.46$

$-1.56$

$-1.75$

$-3.07$

$$
-4.97
$$$$
-3.30
$$$$
-2.73
$$$$
-2.67
$$$$
-3.87
$$$$
0.62
$$$$
1.22
$$$$
0.93
$$$$
5.38
$$$$
5.85
$$$$
0.64
$$$$
1.52
$$

4.12

2.99

3.66

3.56

3.36

3.11

2.89

2.71

2.27

2.12

2.09

2.08

2.02

2.00

2.08

1.40

1.57 
Table 1 Chromosomal genes differentially expressed in response to anaerobic conditions not previously published in E. coli and S. flexneri microarray analysis (Continued)

\begin{tabular}{|c|c|c|c|c|c|}
\hline \multicolumn{2}{|l|}{ SF5M90T_2359 } & \multirow{2}{*}{$\begin{array}{l}\text { beta-fructosidase } \\
\text { triosephosphate isomerase }\end{array}$} & \multicolumn{2}{|l|}{1.49} & \multirow[t]{2}{*}{0.72} \\
\hline SF5M90T_3496 & tpiA & & 1.45 & & \\
\hline SF5M90T_2405 & ptsl & PEP-protein phosphotransferase system enzyme I & 1.42 & & \\
\hline SF5M90T_2808 & fucl & L-fucose isomerase & 1.41 & & \\
\hline SF5M90T_2875 & $b g / A$ & 6-phospho-beta-glucosidase A & 1.27 & & \\
\hline SF5M90T_3348 & malP & maltodextrin phosphorylase & 1.16 & & 0.78 \\
\hline SF5M90T_1107 & ycfO & beta-hexosaminidase & 1.13 & & \\
\hline SF5M90T_8 & talB & transaldolase B & 1.10 & & \\
\hline SF5M90T_2033 & gnd & gluconate-6-phosphate dehydrogenase & 1.01 & & \\
\hline SF5M90T_581 & galm & galactose-1-epimerase & 1.01 & 1.41 & 1.00 \\
\hline SF5M90T_1805 & eda & $\begin{array}{l}\text { keto-hydroxyglutarate-aldolase/keto-deoxy-phosphogluconate } \\
\text { aldolase }\end{array}$ & 0.97 & & \\
\hline SF5M90T_580 & galk & galactokinase & 0.95 & & 0.62 \\
\hline SF5M90T_2913 & $t k t A$ & transketolase 1 isozyme & 0.80 & & \\
\hline SF5M90T_2187 & fruB & PTS system fructose-specific transporter subunit IIA/HPr protein & -1.33 & & \\
\hline SF5M90T_2186 & fruk & fructose-1-phosphate kinase & -1.75 & & \\
\hline SF5M90T_3161 & ptsO & phosphocarrier protein NPr & -1.76 & & \\
\hline SF5M90T_1637 & & putative transport protein & -1.93 & 1.58 & \\
\hline SF5M90T_2185 & fruA & PTS system, fructose-specific transport protein & -1.99 & & \\
\hline \multicolumn{6}{|c|}{ Aminoacid transport and metabolism } \\
\hline SF5M90T_2823 & $\arg A$ & $\mathrm{~N}$-acetylglutamate synthase & 1.94 & & \\
\hline SF5M90T_1910 & fliY & putative periplasmic binding transport protein & 1.80 & & \\
\hline SF5M90T_625 & ybgh & peptide transporter & 1.64 & -1.60 & -1.21 \\
\hline SF5M90T_292 & pepD & aminoacyl-histidine dipeptidase (peptidase D) & 1.54 & 1.78 & 1.75 \\
\hline SF5M90T_2879 & $g c v T$ & aminomethyltransferase & 1.53 & & 1.44 \\
\hline SF5M90T_284 & proA & gamma-glutamylphosphate reductase & 1.48 & & \\
\hline SF5M90T_1121 & potD & spermidine/putrescine periplasmic transport protein & 1.44 & & -0.69 \\
\hline SF5M90T_2674 & cys $D$ & ATP:sulfurylase (ATP:sulfate adenylyltransferase), subunit 2 & 1.39 & -2.30 & -2.21 \\
\hline SF5M90T_1514 & $d c p$ & dipeptidyl carboxypeptidase ॥ & 1.35 & & 0.63 \\
\hline SF5M90T_285 & proB & gamma-glutamate kinase & 1.26 & & -0.58 \\
\hline SF5M90T_2533 & glyA & serine hydroxymethyltransferase & 1.16 & & \\
\hline SF5M90T_2967 & gsp & glutathionylspermidine synthetase/amidase & 1.16 & & 0.70 \\
\hline SF5M90T_1806 & edd & 6-phosphogluconate dehydratase & 1.15 & & -0.72 \\
\hline SF5M90T_807 & & glutathione transporter ATP-binding protein & 1.08 & & \\
\hline SF5M90T_2317 & hisJ & $\begin{array}{l}\text { histidine-binding periplasmic protein of high-affinity } \\
\text { histidine transport system }\end{array}$ & 1.05 & & \\
\hline SF5M90T_806 & ybik & putative asparaginase & 1.02 & & \\
\hline SF5M90T_1122 & potc & spermidine/putrescine transport system permease & 0.97 & -1.09 & -0.92 \\
\hline SF5M90T_2882 & pepP & proline aminopeptidase P ॥ & 0.94 & & \\
\hline SF5M90T_2877 & $g \subset v P$ & glycine decarboxylase & 0.90 & 2.13 & 1.69 \\
\hline SF5M90T_3687 & asnA & asparagine synthetase $A$ & -1.23 & & -1.61 \\
\hline SF5M90T_4099 & lysC & aspartokinase III, lysine sensitive & -1.33 & 1.37 & 1.14 \\
\hline SF5M90T_1253 & $\operatorname{trpE}$ & anthranilate synthase component I & -1.57 & & \\
\hline SF5M90T_4187 & $c y c A$ & transport of D-alanine, D-serine, and glycine & -1.69 & & \\
\hline SF5M90T_1946 & yedA & putative transmembrane subunit & -1.79 & & \\
\hline
\end{tabular}


Table 1 Chromosomal genes differentially expressed in response to anaerobic conditions not previously published in E. coli and S. flexneri microarray analysis (Continued)

\begin{tabular}{lllll}
\hline SF5M90T_3626 & yifK & putative amino acid/amine transport protein & $\mathbf{- 1 . 9 4}$ & \\
SF5M90T_3385 & livJ & Leu/lle/Nal-binding protein precursor & $\mathbf{- 1 . 9 5}$ & $\mathbf{2 . 4}$ \\
SF5M90T_2843 & lysA & diaminopimelate decarboxylase & $\mathbf{- 2 . 1 1}$ & $\mathbf{- 2 . 6 5}$ \\
SF5M90T_4029 & proP & low-affinity transport system; proline permease II & $\mathbf{- 3 . 7 0}$ & $\mathbf{2 . 7}$ \\
SF5M90T_4185 & ytfF & putative transmembrane subunit &
\end{tabular}

SF5M90T_3587 udp uridine phosphorylase

SF5M90T_2456 purC phosphoribosylaminoimidazole-succinocarboxamidesynthetase

Coenzyme transport and metabolism

\begin{tabular}{|c|c|c|}
\hline SF5M90T_2274 & menB & dihydroxynaphtoic acid synthetase \\
\hline SF5M90T_2687 & & phenylacrylic acid decarboxylase-like protein \\
\hline SF5M90T_2276 & menD & 2-oxoglutarate decarboxylase \\
\hline SF5M90T_2273 & menc & O-succinylbenzoate synthase \\
\hline SF5M90T_3142 & $i s p B$ & octaprenyl diphosphate synthase \\
\hline SF5M90T_1613 & $p d x H$ & pyridoxinephosphate oxidase \\
\hline SF5M90T_2880 & visC & putative FAD-dependent oxidoreductase \\
\hline SF5M90T_3011 & $r i b B$ & 3,4 dihydroxy-2-butanone-4-phosphate synthase \\
\hline SF5M90T_3577 & yigc & putative oxidoreductase \\
\hline SF5M90T_2885 & $y g f A$ & putative ligase \\
\hline SF5M90T_3957 & birA & biotin-protein ligase \\
\hline SF5M90T_2103 & thim & hydoxyethylthiazole kinase \\
\hline
\end{tabular}

Lipid transport and metabolism

$\begin{array}{lll}\text { SF5M90T_1094 } & \text { acpP } & \text { acyl carrier protein } \\ \text { SF5M90T_2272 } & \text { menE } & \text { o-succinylbenzoate-CoA ligase } \\ \text { SF5M90T_2416 } & \text { ucpA } & \text { putative oxidoreductase } \\ \text { SF5M90T_339 } & \text { sbmA } & \text { sensitivity to microcin B17, possibly envelope protein }\end{array}$

Inorganic ion transport and metabolism

\begin{tabular}{|c|c|c|}
\hline SF5M90T_2903 & & hypothetical lipoprotein \\
\hline SF5M90T_929 & $y c b O$ & alkanesulfonate transporter substrate-binding subunit \\
\hline SF5M90T_2415 & cys $P$ & thiosulfate binding protein \\
\hline SF5M90T_1636 & $\operatorname{sod} B$ & superoxide dismutase \\
\hline SF5M90T_1187 & & putative ATP-binding protein of $A B C$ transporter \\
\hline SF5M90T_454 & copA & copper exporting ATPase \\
\hline SF5M90T_1186 & & putative iron compound $A B C$ transporter permease \\
\hline SF5M90T_1185 & & iron $A B C$ transporter ATP-binding protein \\
\hline
\end{tabular}


Table 1 Chromosomal genes differentially expressed in response to anaerobic conditions not previously published in E. coli and S. flexneri microarray analysis (Continued)

\begin{tabular}{|c|c|c|c|c|c|}
\hline SF5M90T_4057 & yjcE & predicted cation/proton antiporter & 1.49 & & \\
\hline SF5M90T_2675 & cys $N$ & ATP-sulfurylase (ATP:sulfate adenylyltransferase), subunit 1 & 1.08 & -2.08 & -2.05 \\
\hline SF5M90T_448 & ybal & putative transport protein & -1.01 & & -0.50 \\
\hline SF5M90T_2386 & $m n t H$ & divalent metal cation transporter & -1.93 & 1.61 & 1.68 \\
\hline SF5M90T_330 & tauc & taurine transport system permease protein & -2.09 & & \\
\hline SF5M90T_3769 & shif & putative membrane transport protein & -2.16 & & \\
\hline SF5M90T_3054 & ygjt & putative transport protein & -2.41 & & \\
\hline SF5M90T_1102 & fhuE & outer membrane receptor for ferric iron uptake & -2.46 & & \\
\hline SF5M90T_1483 & ydie & hemin uptake protein & -2.93 & -2.20 & -1.55 \\
\hline SF5M90T_1572 & $m d t$ & spermidine export protein & -3.61 & & \\
\hline \multicolumn{6}{|c|}{ Secondary metabolites biosynthesis, transport and catabolism } \\
\hline SF5M90T_1184 & & putative SAM-dependent methyltransferase & 2.12 & & \\
\hline SF5M90T_331 & taud & taurine dioxygenase, 2-oxoglutarate-dependent & -2.97 & 1.94 & \\
\hline \multicolumn{6}{|c|}{ Cellular processes and signalling } \\
\hline \multicolumn{6}{|c|}{ Cell cycle control, cell division, chromosome partitioning } \\
\hline SF5M90T_1243 & yciB & probable intracellular septation protein A & 0.93 & & -0.80 \\
\hline \multicolumn{6}{|c|}{ Defense mechanisms } \\
\hline SF5M90T_4215 & ampC & beta-lactamase; penicillin resistance & 1.55 & & -1.69 \\
\hline SF5M90T_3751 & emrD & multidrug resistance protein $\mathrm{D}$ & 1.41 & & \\
\hline SF5M90T_4273 & & putative restriction modification enzyme $\mathrm{R}$ subunit & 1.41 & -0.94 & -0.99 \\
\hline SF5M90T_3781 & shiA & virulence factor & 1.30 & & \\
\hline SF5M90T_101 & $a m p D$ & $\mathrm{~N}$-acetyl-anhydromuranmyl-L-alanine amidase & 1.18 & & \\
\hline SF5M90T_772 & ybhF & putative $A B C$-type multidrug transport system component & 1.16 & & 0.54 \\
\hline SF5M90T_771 & ybhs & putative $A B C$-type multidrug transport system component & 1.15 & & \\
\hline SF5M90T_770 & ybhR & putative $A B C$-type multidrug transport system component & 0.90 & & \\
\hline SF5M90T_418 & mdlA & ATP-binding component of a transport system & -1.29 & & \\
\hline \multicolumn{6}{|c|}{ Signal transduction mechanisms } \\
\hline SF5M90T_2126 & yehU & putative 2-component sensor protein & 1.36 & & \\
\hline SF5M90T_3428 & uspA & universal stress protein & 0.86 & & \\
\hline SF5M90T_2388 & yfeA & predicted diguanylate cyclase & -1.20 & & -0.81 \\
\hline SF5M90T_4339 & crec & sensory histidine kinase & -1.63 & 1.43 & \\
\hline \multicolumn{6}{|c|}{ Cell wall/membrane/envelope biogenesis } \\
\hline SF5M90T_1923 & $n m p C$ & outer membrane porin protein & 2.04 & -1.34 & -1.13 \\
\hline SF5M90T_1618 & slyB & putative outer membrane protein & 1.82 & -1.43 & -0.92 \\
\hline SF5M90T_952 & ompA & outer membrane protein $3 a$ & 1.59 & & \\
\hline SF5M90T_374 & $\operatorname{ts} x$ & outer membrane protein & 1.53 & & \\
\hline SF5M90T_256 & gtr $B$ & bactoprenol glucosyl transferase & 1.36 & -2.59 & \\
\hline SF5M90T_2039 & $r f b C$ & dTDP-4-dehydrorhamnose 3,5-epimerase & 1.20 & -2.64 & \\
\hline SF5M90T_4332 & slt & soluble lytic murein transglycosylase & 0.96 & 1.54 & 1.46 \\
\hline SF5M90T_3951 & murl & glutamate racemase & 0.93 & & -0.48 \\
\hline SF5M90T_3821 & $r f a D$ & ADP-L-glycero-D-mannoheptose-6-epimerase & 0.82 & & \\
\hline SF5M90T_1241 & $\operatorname{ton} B$ & transport protein & -1.72 & & \\
\hline SF5M90T_3956 & murB & UDP-N-acetylenolpyruvoylglucosamine reductase & -2.23 & & -0.60 \\
\hline
\end{tabular}


Table 1 Chromosomal genes differentially expressed in response to anaerobic conditions not previously published in E. coli and S. flexneri microarray analysis (Continued)

Cell motility

SF5M90T_1938_fliQ flagellar biosynthetic protein

Intracellular trafficking, secretion and vesicular transport

$\begin{array}{lllr}\text { SF5M90T_3964 } & \text { SecE } & \text { preprotein translocase } & \mathbf{0 . 8 7} \\ \text { SF5M90T_3580 } & \text { tatC } & \text { Sec-independent protein translocase } & \mathbf{0 . 8 4} \\ \text { SF5M90T_3501 } & \text { yiiO } & \text { uncharacterized periplasmic protein } & \mathbf{- 4 . 7 2} \\ \text { Posttranslational } & \text { modification, protein turnover, chaperones } & \\ \text { SF5M90T_4204 } & \text { mopB } & \text { co-chaperonin GroES } & \mathbf{1 . 4 1} \\ \text { SF5M90T_3279 } & \text { SlyD } & \text { FKBP-type peptidyl-prolyl cis-trans isomerase } & \mathbf{1 . 3 1} \\ \text { SF5M90T_462 } & \text { ybbN } & \text { putative thioredoxin-like protein } & \mathbf{0 . 9 0} \\ \text { SF5M90T_407 } & \text { clpP } & \text { ATP-dependent proteolytic subunit of clpA-clpP serine protease } & \mathbf{0 . 8 4} \\ \text { SF5M90T_3738 } & \text { ibpA } & \text { heat shock protein } & \mathbf{- 1 . 1 7} \\ \text { SF5M90T_2074 } & \text { yegD } & \text { putative heat shock protein } & \mathbf{- 2 . 8 0}\end{array}$

0.87

0.84

4.72

1.41

1.31

0.90

0.84

$-1.17$

$-2.80$

\section{Information storage and processing}

Translation, ribosomal structure and biogenesis

$\begin{array}{lcl}\text { SF5M90T_2801 } & \text { yfiA } & \text { translation inhibitor protein RaiA } \\ \text { SF5M90T_2392 } & \text { gltX } & \text { glutamate tRNA synthetase, catalytic subunit } \\ \text { SF5M90T_155 } & \text { frr } & \text { ribosome releasing factor } \\ \text { SF5M90T_650 } & \text { glnS } & \text { glutamine tRNA synthetase } \\ \text { SF5M90T_3893 } & \text { glyQ } & \text { glycine tRNA synthetase, alpha subunit } \\ \text { SF5M90T_4220 } & \text { yjeA } & \text { putative lysyl-tRNA synthetase } \\ \text { SF5M90T_3894 } & \text { glyS } & \text { glycine tRNA synthetase, beta subunit } \\ \text { Transcription } & & \\ \text { SF5M90T_3025 } & \text { ygiP } & \text { putative transcriptional regulator/nucleoid-associated protein } \\ \text { SF5M90T_2417 } & \text { murR } & \text { HTH-type transcriptional regulator } \\ \text { SF5M90T_3510 } & \text { rhaR } & \text { positive regulator for rhaRS operon } \\ \text { SF5M90T_1595 } & \text { mall } & \text { repressor of malX } \text { and Y genes } \\ \text { SF5M90T_2125 } & \text { yehT } & \text { putative two-component response regulator } \\ \text { SF5M90T_1373 } & \text { cSpC } & \text { cold shock protein } \\ \text { SF5M90T_3349 } & \text { malT } & \text { positive regulator of mal regulon } \\ \text { SF5M90T_3335 } & \text { ompR } & \text { osmolarity response regulator } \\ \text { SF5M90T_3453 } & \text { yiaG } & \text { putative transcriptional regulator } \\ \text { SF5M90T_2089 } & \text { gatR } & \text { galactitol utilization operon repressor } \\ \text { SF5M90T_71 } & \text { cra } & \text { transcriptional repressor of fru operon and others } \\ \text { SF5M90T_4197 } & \text { yjdC } & \text { putative transcriptional regulator } \\ \text { SF5M90T_1370 } & & \text { putative regulator } \\ \text { SF5M90T_3578 } & \text { rfaH } & \text { transcriptional activator } \\ \text { SF5M90T_4242 } & \text { yjeB } & \text { HTH-type transcriptional repressor } \\ \text { SF5M90T_984 } & \text { cspH } & \text { cold shock-like protein }\end{array}$

2.70

1.65

1.21

1.08

1.06

0.95

$-1.75$

$-0.50$

0.81

3.04

$-4.57$

$-2.45$

2.35

2.33

1.98

1.80

1.59

$-1.38$

1.58

1.42

1.38

1.33

1.16

1.15

1.37

1.09

$-1.57$

$-0.96$

$-1.95$

$-3.35$

1.42

$-3.26$

$-2.74$

1.23

1.10

SF5M90T_3034

ygjF G/U mismatch-specific DNA glycosylase

1.08

$\begin{array}{lcl}\text { SF5M90T_775 } & \text { rhIE } & \text { putative ATP-dependent RNA helicase }\end{array}$ 
Table 1 Chromosomal genes differentially expressed in response to anaerobic conditions not previously published in E. coli and S. flexneri microarray analysis (Continued)

\begin{tabular}{llll}
\hline SF5M90T_3117 & deaD & inducible ATP-independent RNA helicase & $\mathbf{- 1 . 2 0}$ \\
SF5M90T_1769 & $d b p A$ & ATP-dependent RNA helicase & $\mathbf{- 1 . 8 6}$
\end{tabular}

\section{Poorly characterized}

General function prediction only

\begin{tabular}{|c|c|c|c|c|c|}
\hline SF5M90T_2762 & $\operatorname{stp} A$ & DNA-binding protein & 3.51 & -3.00 & -2.60 \\
\hline SF5M90T_275 & & putative crossover junction endodeoxyribonuclease & 2.84 & & \\
\hline SF5M90T_2418 & muQ & $\mathrm{N}$-acetylmuramic acid 6-phosphate etherase & 2.77 & & -0.93 \\
\hline SF5M90T_1724 & & putative acetyltransferase & 2.06 & -2.41 & \\
\hline SF5M90T_2435 & & putative amino acid antiporter & 2.03 & 1.90 & 1.58 \\
\hline SF5M90T_2301 & $y f b T$ & putative phosphatase & 2.00 & & \\
\hline SF5M90T_773 & ybhG & putative membrane protein & 1.78 & 1.02 & \\
\hline SF5M90T_1227 & hns & DNA-binding protein & 1.69 & -1.24 & \\
\hline SF5M90T_2275 & $y f b B$ & putative enzyme & 1.62 & -1.90 & -1.85 \\
\hline SF5M90T_3225 & $y r d A$ & putative transferase & 1.51 & & \\
\hline SF5M90T_4236 & $h f q$ & RNA-binding protein & 1.45 & -2.10 & \\
\hline SF5M90T_3315 & gph & phosphoglycolate phosphatase & 1.25 & & \\
\hline SF5M90T_2192 & yeir & putative GTPases & 1.03 & & \\
\hline SF5M90T_1919 & yedE & putative transport system permease protein & 1.03 & -1.83 & -1.96 \\
\hline SF5M90T_3295 & yhfC & putative transport & 0.97 & -1.30 & -1.25 \\
\hline SF5M90T_2205 & yejk & nucleoid-associated protein & 0.95 & & \\
\hline SF5M90T_2066 & yegH & putative transport protein & 0.84 & & \\
\hline SF5M90T_3344 & yhgH & putative gluconate periplasmic binding protein & 0.81 & & \\
\hline SF5M90T_3102 & yraM & putative glycosylase & 0.73 & & \\
\hline SF5M90T_794 & ybip & putative enzyme & -1.14 & & \\
\hline SF5M90T_2207 & уејм & putative sulfatase & -1.36 & & \\
\hline SF5M90T_3139 & $y h b E$ & $\begin{array}{l}\text { putative permeases of drug/metabolite } \\
\text { transporter superfamily }\end{array}$ & -1.36 & & -0.90 \\
\hline SF5M90T_966 & $y \subset C A$ & putative carrier/transport protein & -1.38 & & -1.18 \\
\hline SF5M90T_2742 & yqaB & putative phosphatase & -1.51 & & \\
\hline SF5M90T_3882 & bax & putative ATP-binding protein & -1.70 & & -0.77 \\
\hline SF5M90T_3370 & $y h h x$ & putative regulator & -1.97 & & 1.30 \\
\hline SF5M90T_3621 & $a s / B$ & putative arylsulfatase regulator & -2.73 & & \\
\hline SF5M90T_2516 & & putative enzyme & -3.91 & & \\
\hline \multicolumn{6}{|c|}{ Function unknown } \\
\hline SFxv_3833 & & conserved hypothetical protein & 3.59 & -4.23 & \\
\hline SF5M90T_2431 & & conserved hypothetical protein & 2.89 & & \\
\hline SF5M90T_2432 & & conserved hypothetical protein & 2.69 & & \\
\hline SF5M90T_11 & & uncharacterized protein & 2.57 & & \\
\hline SF5M90T_1402 & yead & conserved hypothetical protein & 2.45 & & \\
\hline SF5M90T_828 & ybjo & conserved hypothetical protein & 2.23 & & -2.52 \\
\hline SF5M90T_1941 & $d s r B$ & conserved hypothetical protein & 2.03 & -2.43 & \\
\hline SF5M90T_2302 & $y f b U$ & conserved hypothetical protein & 1.87 & & \\
\hline SF5M90T_451 & ybak & conserved hypothetical protein & 1.86 & -1.21 & -1.10 \\
\hline SSJG_00311 & & conserved hypothetical protein & 1.75 & & -1.60 \\
\hline
\end{tabular}


Table 1 Chromosomal genes differentially expressed in response to anaerobic conditions not previously published in E. coli and S. flexneri microarray analysis (Continued)

\begin{tabular}{|c|c|c|c|c|c|}
\hline SF5M90T_5 & yaaA & conserved hypothetical protein & 1.54 & & \\
\hline SF5M90T_1387 & & conserved hypothetical protein & 1.51 & & \\
\hline SF5M90T_3911 & yiil & conserved hypothetical protein & 1.45 & -2.49 & \\
\hline SF5M90T_957 & & conserved hypothetical protein & 1.37 & & \\
\hline SF5M90T_4146 & yjgD & conserved hypothetical protein & 1.28 & -2.30 & \\
\hline SF5M90T_2622 & & conserved hypothetical protein & 1.24 & -1.46 & \\
\hline SF5M90T_3155 & $y h b N$ & conserved hypothetical protein & 0.81 & & \\
\hline SF5M90T_479 & $y b b F$ & conserved hypothetical protein & -1.19 & & \\
\hline SF5M90T_2195 & $r t n$ & conserved hypothetical protein & -1.50 & & \\
\hline SF5M90T_438 & ybaN & conserved hypothetical protein & -1.73 & & \\
\hline SF5M90T_1853 & & conserved hypothetical protein & -2.03 & -2.58 & \\
\hline SF5M90T_1647 & & conserved hypothetical protein & -2.11 & & \\
\hline SF5M90T_983 & $y m c D$ & conserved hypothetical protein & -2.34 & & \\
\hline SF5M90T_4094 & $y j b A$ & P-starvation inducible protein PsiE & -2.51 & 1.69 & \\
\hline SF5M90T_1110 & $y(f)$ & conserved hypothetical protein & -2.52 & & 0.99 \\
\hline SF2861 & & hypothetical protein remnant & -2.64 & & \\
\hline SF5M90T_2146 & yoho & membrane protein & -2.96 & & \\
\hline SF5M90T_1952 & & putative outer membrane pore protein & -2.98 & & \\
\hline SF5M90T_4307 & & putative inner membrane protein & -3.40 & & \\
\hline SF1231 & & conserved hypothetical protein & -3.71 & & -1.60 \\
\hline SF5M90T_427 & ybaA & conserved hypothetical protein & -3.88 & & \\
\hline \multicolumn{6}{|l|}{ Phage related } \\
\hline S1668 & relF & prophage maintenance protein & 1.75 & & \\
\hline SF5M90T_1793 & & putative phage integrase protein & 1.45 & -1.60 & \\
\hline SF5M90T_1056 & & hypothetical bacteriophage protein & 1.14 & & \\
\hline SF5M90T_740 & & putative bacteriophage protein & -1.93 & & \\
\hline
\end{tabular}

${ }^{a}$ Genomes used as reference are: S. flexneri 5a str. M90T, S. flexneri 2a str. 301, S. flexneri 2002017, Shigella sp. D9 and S. flexneri 2457 T with GenBank accession numbers AGNM00000000, NC 004337, NC 017328, NZ GG657384 and NC_004741 respectively.

${ }^{\mathrm{b}}$ Genes are classified in functional categories based on the database of Clusters of Orthologous Groups (COGs). http://www.ncbi.nlm.nih.gov/COG/. Inside each subgroup, genes are arranged in descending order in relation to Log2 of Fold Change values of WT no $\mathrm{O}_{2} / \mathrm{WT} \mathrm{O}_{2}$ comparison.

'Log2 of Fold Change values of WT no $\mathrm{O}_{2} / \mathrm{WT} \mathrm{O}_{2}$ and $\Delta f n r$ no $\mathrm{O}_{2} / \mathrm{WT}$ no $\mathrm{O}_{2}$ comparisons are presented. Only values considered differentially expressed are shown ( $p$ adjust $<0.05$ ).

also examined the transcription profile of parB [65]. Similar to parA, mRNA levels of parB are elevated during anaerobic growth (Figure 5). Consistent with this finding, there was no significant difference in loss of the virulence plasmid from bacteria grown in aerobic and anaerobic conditions (not shown).

The Shigella pathogenicity island SHI-1 is not present in S. flexneri M90T. Therefore, we examined the transcriptional profile of the SHI-2 pathogenicity island that includes the aerobactin, iron-uptake system [66]. As previously reported, we found that genes encoding the aerobactin system (iucABCD and $i u t A$ ) were down-regulated under anaerobic conditions, as was shiF, a gene which is also involved in iron acquisition [6,67]. In contrast, shiA, a SHI-2 gene involved in attenuating host inflammatory responses, was overexpressed under anaerobic conditions when compared to aerobic conditions [68]. Of note, no SHI-2 gene is subject to FNR regulation (Table 1, Figure 5, see Additional file 1: Table S1).

csrB and csrC sRNAs are induced in the absence of oxygen in S. flexneri M90T

Little is known about the small RNAs (sRNAs) in Shigella or their expression under anaerobic conditions. We analysed the sRNAs already described in Shigella as well as potential sRNAs homologues to those described in S. enterica serovar Typhimurium and found that anaerobic growth conditions induce the expression of $\mathrm{csrB}$ and $\operatorname{csrC}$ in an FNR-independent manner (Table 3, Figure 6) [69-71]. 


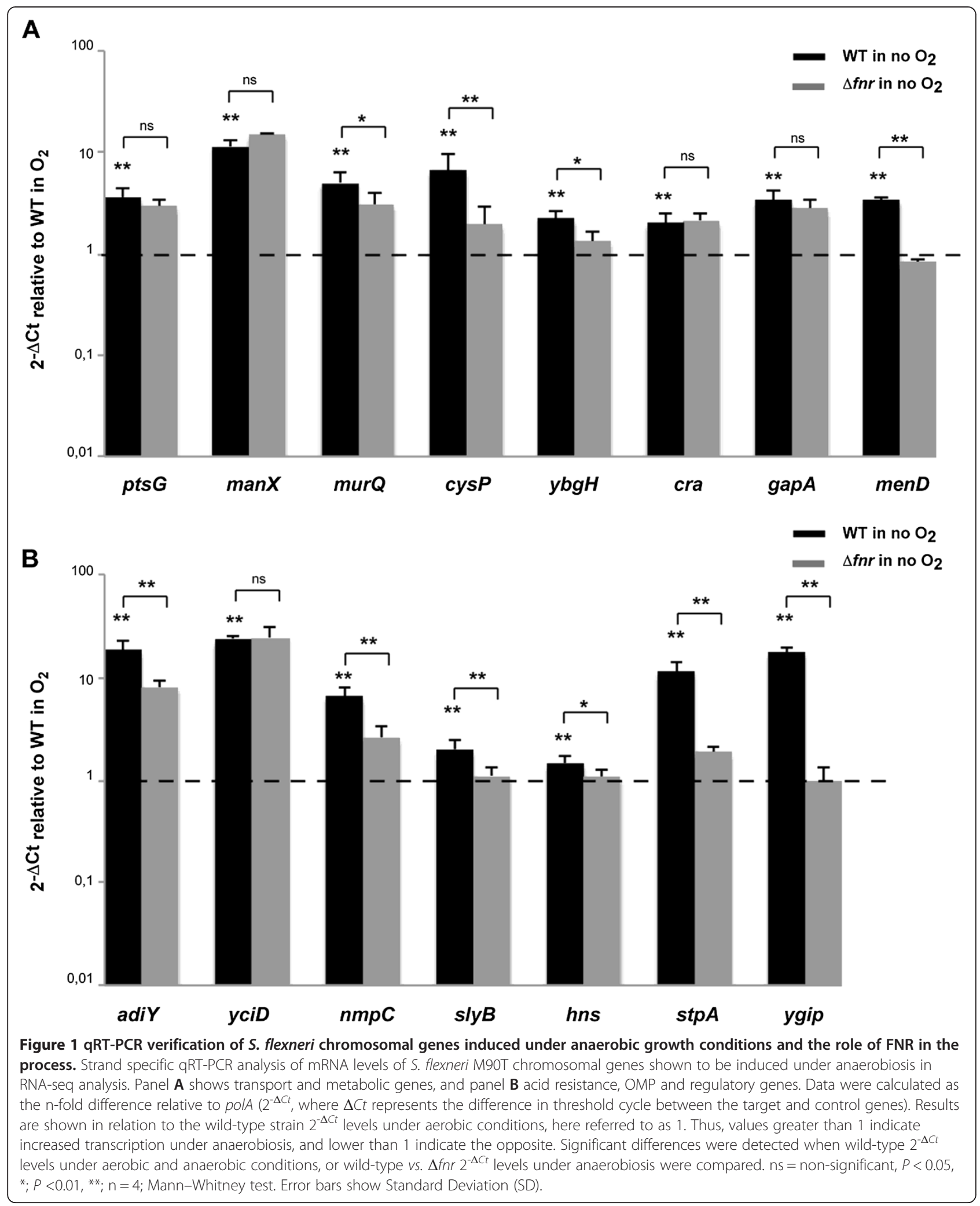

\section{Discussion}

In vitro studies have several limitations in relation to in vivo studies; e.g., they cannot mimic the amount and type of carbon sources available for bacteria and lack the signals derived from the interaction with intestinal epithelium, human immune system or other bacteria 


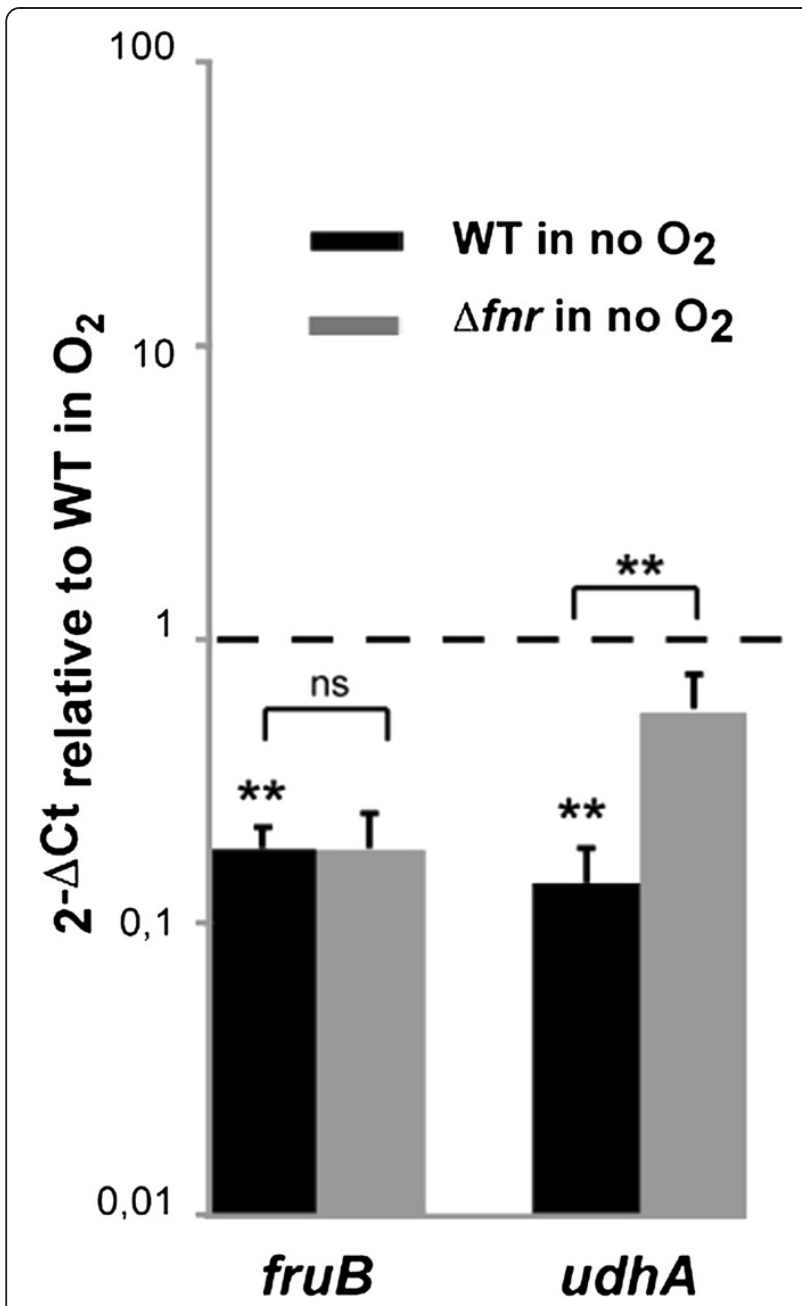

Figure 2 qRT-PCR verification of S. flexneri chromosomal genes repressed under anaerobic growth conditions and the role of FNR in the repression. Strand specific qRT-PCR analysis of mRNA levels of S. flexneri M90T chromosomal genes shown to be repressed in RNA-seq analysis. Data were calculated as the $\mathrm{n}$-fold difference relative to polA ( $2^{-\Delta C t}$, where $\Delta C$ t represents the difference in threshold cycle between the target and control genes). Results are shown in relation to wild-type $2^{-\Delta C t}$ levels under aerobic conditions, here referred to as 1 . Thus, values greater than 1 indicate increased transcription under anaerobiosis and lower than 1 indicates the opposite. Significant differences were detected when wild-type $2^{-\Delta c t}$ levels under aerobic and anaerobic conditions or wild-type vs. $\Delta f n r 2^{-\Delta C t}$ levels under anaerobiosis were compared. $P<0.01,{ }^{* *} ; n=4$; Mann-Whitney test. Error bars show Standard Deviation (SD).

present in the gut. However, if conducted accurately can provide valuable information.

In the current study we have, for the first time, employed RNA-sequencing to identify oxygen regulated genes in an enteric pathogen. Our findings confirm previous results, but as this method is more sensitive than array based approaches, we identified an extended repertoire of genes modulated by oxygen in an FNRdependent or -independent manner. For instance, little is known about the role of Cra, a transcriptional regulator of carbon flux (that represses glycolysis and activates gluconeogenesis) here shown to be induced under anaerobic conditions [41]. Interestingly, mutation of cra increases both epithelial cell attachment and invasion by Shigella in aerobic conditions [72]. However, Cra has an entirely distinct role in the virulence of enterohemorrhagic E. coli (EHEC), a close relative of Shigella, when investigated under conditions mimicking the anaerobic environment of the intestinal tract. Under these circumstances, loss of Cra reduces attachment of bacteria to enterocytes [73]. Additionally, Salmonella cra mutants are avirulent when administered orally, indicating that Cra may have key roles in enteric pathogens in anaerobic conditions [74].

While there is an increasing recognition that carbon metabolism affects microbial virulence, it is still not clear whether distinct carbon energy sources are important or preferable for different members of the Enterobacteriaceae [72,75-80]. For example, our results show that the expression pattern under anaerobic conditions of ptsG, $\operatorname{man} X Y C$ and fruBKA involved in the transport of sugars is opposite in Shigella to that observed in $E$. coli $[18,20]$. This could be simply due to the different growth medium used in the experiments or to distinct metabolic strategies between Shigella and other Enterobacteriaceae. In favour of the latter and its relationship with virulence it has been shown that mutation of ptsG induces the adherence and invasive capacity of enteroinvasive E. coli (EIEC) strains but not in Salmonella [81]. Further differences between Shigella and other Enterobacteriaceae include adiY, an AraC-like regulator, which activates expression of adiA and adiC, encoding the arginine-dependent acid resistance system (AR3). In Salmonella adiY expression is elevated under aerobic conditions, whereas in Shigella and in E. coli, increased expression of adiY occurs in anaerobiosis [20,82]. These differences could be due to the strikingly different acid survival strategies that these bacteria seem to develop in spite of being close relatives [83]. Deletion of cad locus, a typical pathoadaptive mutation in Shigella spp., also induces the AR3 system suggesting that this system contributes to the survival of Shigella in its particular niche in the intestinal tract $[84,85]$.

Interestingly, we observed an FNR-dependent elevated expression under anaerobiosis of $h n s$ and overall of $s t p A$ and ygiP that encode nucleoid-associated proteins responsible for DNA compaction and global gene regulation, indicating that lack of oxygen profoundly modifies DNA topology in Shigella. Recently, it has been shown that FNR function is strongly inhibited by this kind of nucleoid-associated proteins, which block FNR access to many binding sites [20]. Our findings suggest that FNR is involved in this inhibition, probably indirectly, due to 
Table 2 Virulence plasmid genes differentially expressed in response to anaerobic conditions

\begin{tabular}{|c|c|c|c|c|c|}
\hline ORF ID & Gene & Description & $\frac{\text { RNA-seq }{ }^{c} \log 2 \mathrm{FC}}{\text { WT no } \mathrm{O}_{2} / \mathrm{O}_{2}}$ & $\frac{\text { RNA-seq }^{\mathrm{c}} \log 2 \mathrm{FC}}{\Delta \text { fnr/WT no } \mathrm{O}_{2}}$ & $\frac{\text { FRT-seq }^{\mathrm{c}} \log 2 \mathrm{FC}}{\Delta \text { fnr/WT no } \mathrm{O}_{2}}$ \\
\hline pWR501_0265 & yigB & hypothetical protein & 2.56 & & \\
\hline pWR501_0225 & ospl & T3SS effector & 2.00 & -2.21 & \\
\hline pWR501_0250 & $\operatorname{shf}$ & peptidoglycan deacetylase & 1.24 & -3.42 & -1.30 \\
\hline pWR501_0251 & $r f b U$ & glycosiltransferase & 1.21 & -1.65 & -1.16 \\
\hline pWR501_0252 & virk & virulence protein & 1.15 & -2.16 & -1.03 \\
\hline pWR501_0039 & parA & plasmid segregation protein & 1.13 & & \\
\hline pWR501_0253 & $m s b B$ & acyltransferase & 1.07 & -3.03 & -1.08 \\
\hline pWR501_0074 & $\operatorname{sep} A$ & secreted protease & -1.12 & 1.34 & 1.52 \\
\hline pWR501_0177 & & hypothetical protein & -1.55 & & 1.04 \\
\hline pWR501_0283 & ipaH1.4 & T3SS effector & -1.56 & & \\
\hline pWR501_0175 & & hypothetical protein & -1.58 & & 2.29 \\
\hline pWR501_0176 & & hypothetical protein & -1.76 & & 2.02 \\
\hline pWR501_0015 & & hypothetical protein & -2.00 & 1.52 & 1.05 \\
\hline pWR501_0002 & & putative resolvase & -2.04 & 1.97 & \\
\hline pWR501_0007 & & hypothetical protein & -2.25 & 1.63 & \\
\hline pWR501_0014 & & hypothetical protein & -2.25 & 1.60 & 1.27 \\
\hline pWR501_0192 & virG & invasion protein & -2.25 & & 2.36 \\
\hline pWR501_0144 & $i p g F$ & unknown function & -2.38 & & 3.20 \\
\hline pWR501_0051 & virf & transcriptional activator of virulence & -2.47 & & \\
\hline pWR501_0006 & & hypothetical protein & -2.54 & & \\
\hline pWR501_0143 & $i p g E$ & chaperon & -2.55 & & 3.39 \\
\hline pWR501_0146 & $m \times i H$ & T3SS component & -2.56 & 1.55 & 4.10 \\
\hline pWR501_0122 & & hypothetical protein & -2.58 & 1.53 & 1.95 \\
\hline pWR501_0121 & & hypothetical protein & -2.65 & & \\
\hline pWR501_0191 & virA & T3SS effector & -2.66 & & 2.42 \\
\hline pWR501_0147 & mxil & T3SS component & -2.80 & 2.00 & 4.01 \\
\hline pWR501_0013 & $m k a D$ & mouse killing factor & -2.81 & 1.97 & 3.33 \\
\hline pWR501_0145 & $m \times i G$ & T3SS component & -3.02 & & 3.45 \\
\hline pWR501_0148 & $m \times i J$ & T3SS component & -3.06 & 2.17 & 4.33 \\
\hline pWR501_0031 & & hypothetical protein & -3.14 & & \\
\hline pWR501_0005 & & hypothetical protein & -3.14 & & 2.49 \\
\hline pWR501_0292 & sopA & VirG-specific protease & -3.25 & & 2.49 \\
\hline pWR501_0291 & & hypothetical protein & -3.31 & & \\
\hline pWR501_0138 & $i p g B$ & invasion protein & -3.34 & & 3.66 \\
\hline pWR501_0157 & spa15 & chaperon & -3.34 & 3.52 & 4.43 \\
\hline pWR501_0012 & ShET2-2 & enterotoxin & -3.38 & & 3.41 \\
\hline pWR501_0156 & $m \times i A$ & T3SS component & -3.44 & & 4.67 \\
\hline pWR501_0160 & spa32 & invasion protein & -3.45 & 1.34 & 4.63 \\
\hline pWR501_0141 & & hypothetical protein & -3.50 & & 3.71 \\
\hline pWR501_0004 & phoN2 & apyrase & -3.52 & 2.38 & 3.25 \\
\hline pWR501_0150 & $m \times i L$ & hypothetical protein & -3.59 & 2.07 & 4.64 \\
\hline pWR501_0132 & $a c p$ & hypothetical protein & -3.62 & & 5.19 \\
\hline pWR501_0166 & spa-orf10 & hypothetical protein & -3.64 & 1.97 & 4.52 \\
\hline
\end{tabular}


Table 2 Virulence plasmid genes differentially expressed in response to anaerobic conditions (Continued)

\begin{tabular}{|c|c|c|c|c|c|}
\hline pWR501_0158 & spa47 & T3SS component & -3.65 & 3.19 & 4.69 \\
\hline pWR501_0140 & $i c s B$ & T3SS effector & -3.65 & 2.16 & 4.43 \\
\hline pWR501_0162 & spa24 & T3SS component & -3.70 & 2.28 & 3.71 \\
\hline pWR501_0159 & spa13 & T3SS component & -3.75 & & 5.13 \\
\hline pWR501_0161 & spa33 & T3SS component & -3.75 & 1.99 & 4.03 \\
\hline pWR501_0151 & $m \times i M$ & T3SS component & -3.81 & 2.40 & 4.25 \\
\hline pWR501_0155 & $m \times i C$ & T3SS component & -3.86 & 2.43 & 4.95 \\
\hline pWR501_0290 & & hypothetical protein & -3.86 & & 2.00 \\
\hline pWR501_0030 & & putative enterotoxin fragment & -3.90 & 2.20 & 4.54 \\
\hline pWR501_0163 & spa9 & T3SS component & -3.92 & 1.93 & 3.56 \\
\hline pWR501_0137 & $i p g C$ & chaperon & -3.93 & 1.86 & 4.04 \\
\hline pWR501_0135 & $i p a C$ & T3SS effector & -3.93 & 2.65 & 5.01 \\
\hline pWR501_0165 & spa40 & T3SS component & -3.94 & & 4.02 \\
\hline pWR501_0139 & ipgA & chaperon & -3.95 & 2.45 & 4.25 \\
\hline pWR501_0153 & $m \times i D$ & T3SS component & -3.98 & 2.33 & 4.81 \\
\hline pWR501_0152 & $m \times i E$ & transcriptional activator & -4.06 & 2.51 & 4.48 \\
\hline pWR501_0154 & $m \times i D$ & T3SS component & -4.08 & 2.66 & 4.54 \\
\hline pWR501_0134 & $i p a D$ & T3SS effector & -4.16 & 2.99 & 4.92 \\
\hline pWR501_0167 & spa-orf11 & hypothetical protein & -4.19 & 2.85 & 4.07 \\
\hline pWR501_0136 & $i p a B$ & T3SS effector & -4.24 & 2.97 & 4.59 \\
\hline pWR501_0133 & $i p a A$ & T3SS effector & -4.24 & 3.09 & 4.98 \\
\hline pWR501_0003 & & hypothetical protein & -4.57 & & 3.29 \\
\hline pWR501_0164 & spa29 & T3SS component & -5.06 & 2.36 & 3.32 \\
\hline pWR501_0131 & $\operatorname{vir} B$ & transcriptional activator & -5.17 & 2.40 & 4.26 \\
\hline
\end{tabular}

${ }^{a}$ S. flexneri 5a str. M90T pWR501 virulence plasmid sequence was used as reference GenBank accession numbers AF348706.

${ }^{\mathrm{b}} \mathrm{Genes}$ are arranged in descending order in relation to Log2 of Fold Change values of WT no $\mathrm{O}_{2} / \mathrm{WT}^{\mathrm{O}_{2}}$ comparison.

${ }^{c}$ Log2 of Fold Change values of WT no $\mathrm{O}_{2} / \mathrm{WT} \mathrm{O}_{2}$ and $\Delta f n r$ no $\mathrm{O}_{2} / \mathrm{WT}$ no $\mathrm{O}_{2}$ comparisons are presented. Only values considered differentially expressed are shown ( $p$ adjust $<0.05$ ).

the absence of putative FNR binding-boxes in the promoter region of these genes [20].

To distinguish between direct and indirect effects of FNR, in vivo approaches based in chromatin immunoprecipitation followed by micro-array hybridization (ChIP-chip) or high-throughput sequencing (ChIP-seq) have been performed in E. coli $[20,86]$. Correlation of FNR ChIP-seq peaks with transcriptomic data showed that less than half of the FNR-regulated operons could be attributed to direct FNR binding. Of note, FNR occupancy does not always correlate with the presence of a consensus FNR binding site or a change in expression $[20,86]$. A total of 19 of E. coli ChIP-seq peaks are located in promoter regions of genes identified in Table 1 (i.e. ptsG, pfkA, gapA, yegT, ptsH, tpiA, lysC, menD, ribB, uspA, slyB, отрA, tonB, yjeA, cspH, deaD, dbpA, yccA and $y h h X$ ); only one of these, $\operatorname{dbpA}$, has a canonical FNR binding sequence in its promoter region. Consistent with previous findings, only six of these 19 genes (lys $C$, menD, slyB, yjeA, $y c c A$ and $y h h X$ ) were influenced by FNR in our transcriptomic analysis. This result suggests that many FNR effects in Table 1 are likely to be indirect. However, we cannot rule out differences in regulation between E. coli and Shigella that could affect FNR function. Of note, this is the first time that menD, slyB, yjeA and $y h h X$ have been identified as FNR regulated by transcriptome analysis, corroborating previous ChIP findings performed in E. coli.

sRNAs are widespread in bacteria and play critical roles in regulating physiological processes [87]. In Shigella, putative sRNAs have been identified by bioinformatics $[69,70]$. However, the expression of these sRNAs has not been confirmed in all cases and little is known about their function or the physiological conditions that induce their expression. Here, we found that anaerobic growth induces expression of two sRNAs, csrB and $\operatorname{csrC}$, independently of FNR. In E. coli $\operatorname{csrB}$ and $\operatorname{csrC}$ regulate the activity of $\mathrm{CsrA}$, the carbon storage regulator although their function in Shigella has not been characterised so far $[88,89]$.

For genes directly involved in host:pathogen interactions, we found that oxygen influences the expression of 


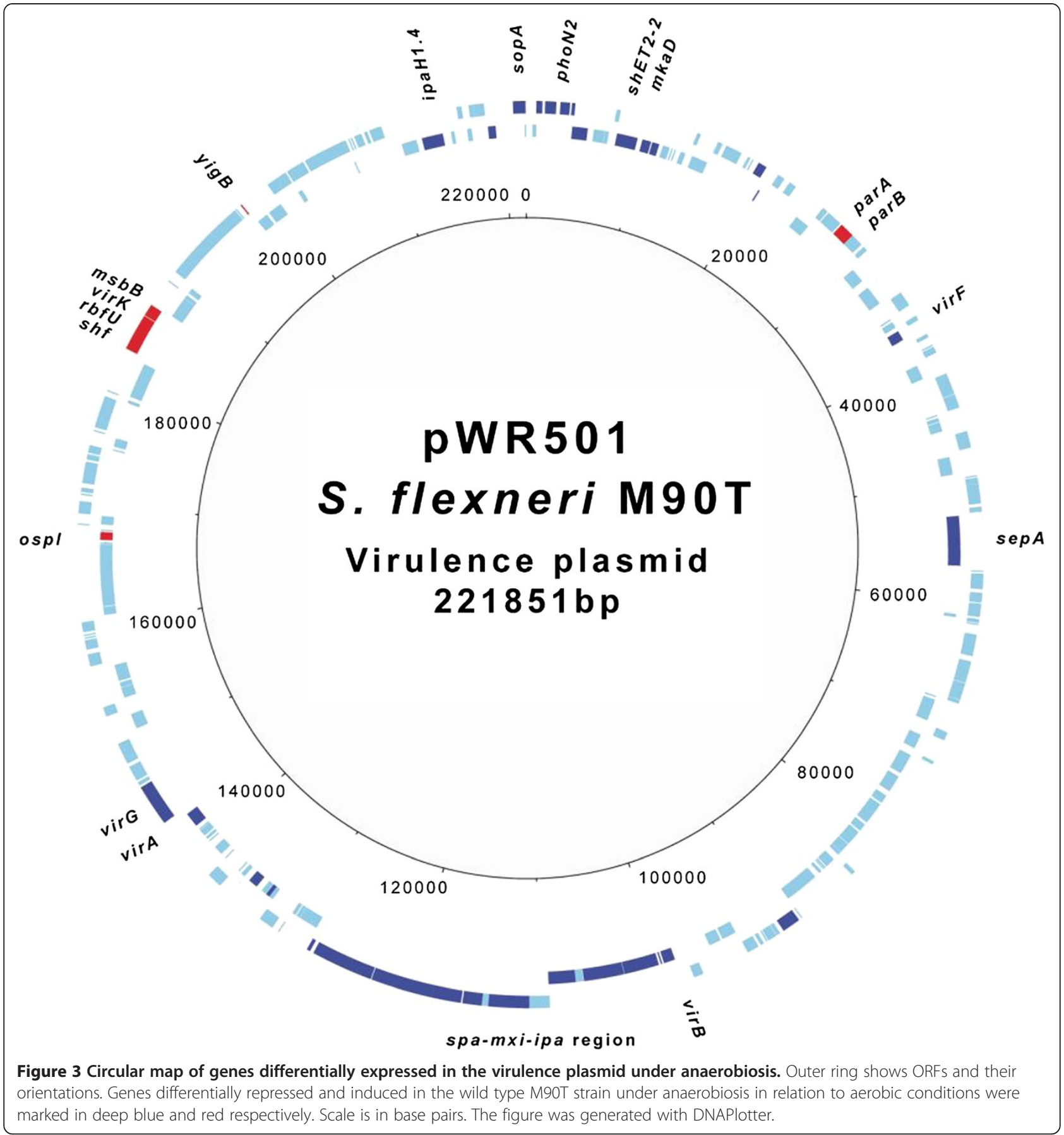

almost all genes in the mxi-spa operon. These T3SSrelated genes were down-regulated in the absence of oxygen in an FNR-dependent manner. This is likely to be mediated by VirB as this transcription factor controls many genes in this operon, is influenced by H-NS dependent DNA supercoiling and our findings demonstrate that virB gene is repressed in anaerobiosis [90]. The effect of oxygen on the Shigella T3SS is opposite to Salmonella in which FNR induces expression of invasion genes, and probably reflects the different sites occupied in the host by these two related intestinal pathogens [19]. The results further emphasise that the Shigella T3SS is inactive in anaerobic environments as we previously reported [7].

Inflammation at the site of invasive infection is a hallmark of intestinal shigellosis $[91,92]$. Of note, expression of shiA is induced under anaerobiosis. This gene in the SHI-2 pathogenicity island encodes a factor that attenuates the intestinal inflammatory response in shigellosis by decreasing the recruitment of polymorphonuclear 

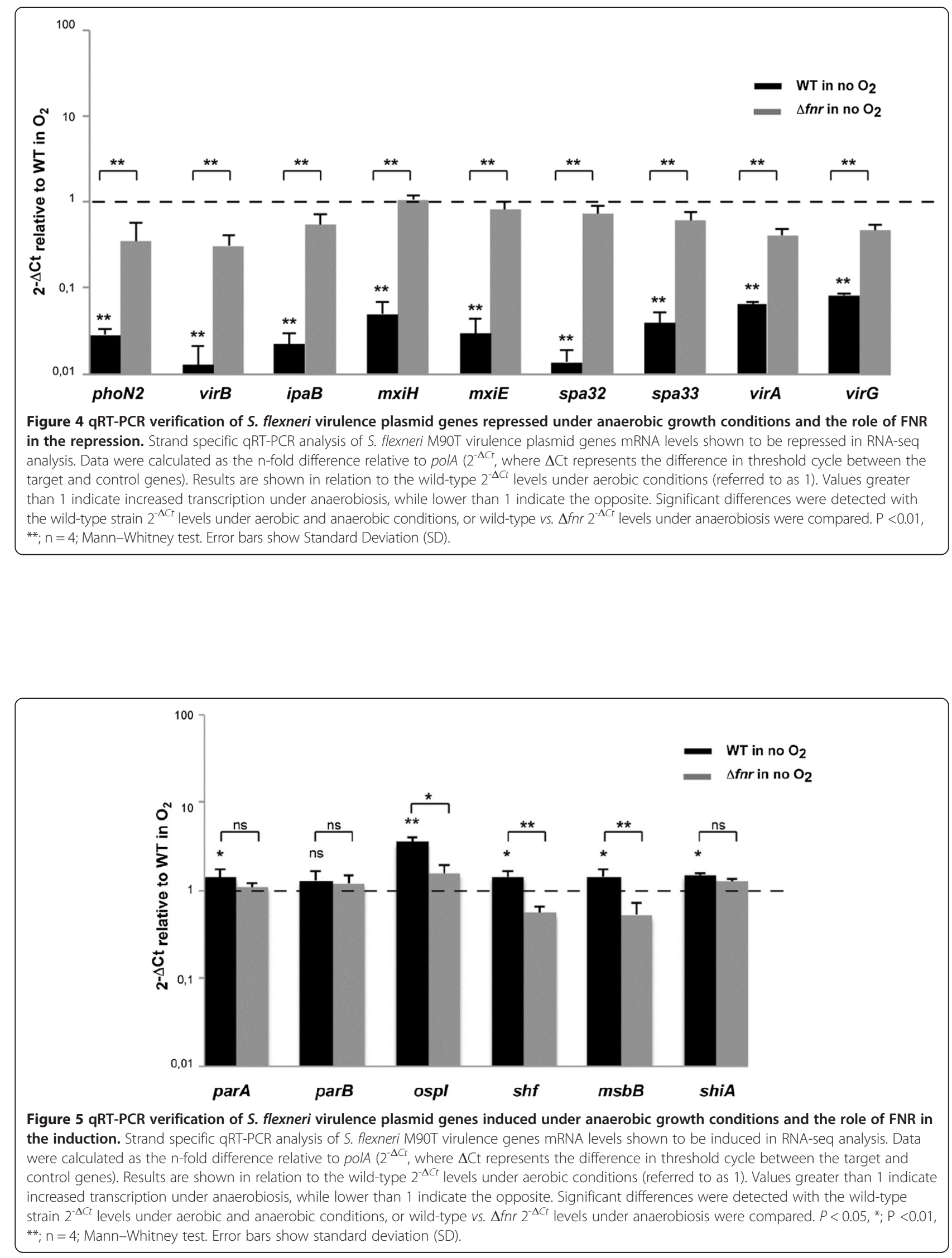
Table 3 sRNAs differentially expressed in response to anaerobic conditions

\begin{tabular}{|c|c|c|c|c|c|}
\hline \multirow[t]{2}{*}{ SRNA $^{a}$} & \multirow[t]{2}{*}{ Adjacent genes } & \multirow[t]{2}{*}{ Description/class } & \multirow{2}{*}{$\begin{array}{c}\text { Length } \\
\text { (nt) }\end{array}$} & \multirow{2}{*}{$\frac{\text { RNA-seq }^{\text {b }} \log 2 \mathrm{FC}}{\text { WT no } \mathrm{O}_{2} / \mathrm{O}_{2}}$} & \multirow{2}{*}{$\frac{\text { RNA-seq }^{\mathrm{b}} \log 2 \mathrm{FC}}{\Delta \text { fnr/WT no } \mathrm{O}_{2}}$} \\
\hline & & & & & \\
\hline $\operatorname{csr} B$ & syd/SF5M90T_2595 & protein-binding sRNA & 360 & 4.97 & \\
\hline $\operatorname{csrC}$ & yihi/yihA & protein-binding sRNA & 245 & 3.38 & \\
\hline
\end{tabular}

${ }^{a}$ sRNAs are arranged in descending order in relation to Log2 of Fold Change values of WT no $\mathrm{O}_{2} / \mathrm{WT}_{2}$ comparison.

${ }^{b} \mathrm{Log} 2$ of Fold Change values of WT no $\mathrm{O}_{2} / \mathrm{WT} \mathrm{O}_{2}$ and $\Delta$ fnr no $\mathrm{O}_{2} / \mathrm{WT}$ no $\mathrm{O}_{2}$ comparisons are presented. Only values considered differentially expressed are shown ( $p$ adjust $<0.05)$.

leukocytes and T-cells [68,93]. Similarly OspI is the only T3SS-effector protein that was overexpressed in anaerobiosis; it also serves to dampen inflammatory responses by deaminating a glutamine in host ubiquitin-conjugating enzyme (UBC13) [94]. Thus, expression of both ShiA and OspI under low oxygen tension might dampen the extent of inflammatory responses to Shigella while it is in the anoxic environment of the intestinal lumen, impairing immune responses. Only one operon on the virulence plasmid, shf-rfbU-virK-msbB, was induced under anaerobiosis in an FNR-dependent manner. Interestingly, all these genes are implicated in modification of Shigella lipopolysaccharide (LPS), an important pro-inflammatory mediator [95-99].

The transcription of several genes encoding OMPs was induced under anaerobic growth. Both OmpA and OmpC have been implicated in Shigella virulence, while our results suggest that Tsx, Slp, NmpC, SlyB and YciD (OmpW) could also contribute to pathogenesis and be considered as potential vaccine targets $[100,101]$. Indeed, Salmonella OmpW, Tsx and NmpC have already been demonstrated to be immunogenic [102,103]. In addition to OMPs, transcription of gapA, which encodes glyceraldehyde-3-phosphate dehydrogenase, was induced under anaerobic conditions. Interestingly, this enzyme is exported by EHEC and enteropathogenic E. coli (EPEC) strains but not by non-pathogenic strains. Due to its ability to interact with plasminogen, fibrinogen and intestinal epithelial cells, it has been suggested that GapA might

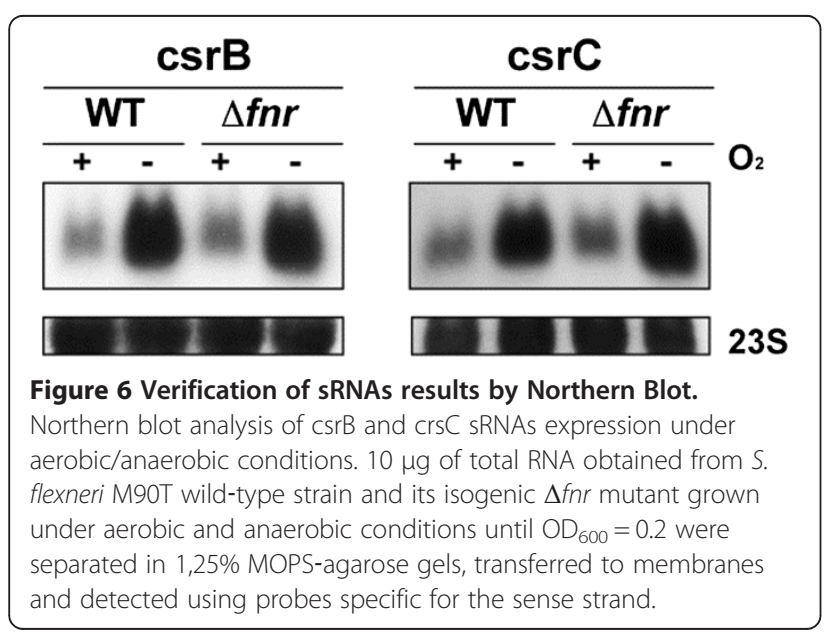

contribute in vivo to the interaction of EHEC and EPEC with the gut epithelium [104].

\section{Conclusions}

Overall, our RNA-seq based analysis revealed that in the anaerobic lumen of the intestine Shigella is predicted to prompt both survival and anti-host immune-modulatory activities of the bacterium. This occurs through a reprogramming of bacterial metabolism including altered transcription of genes encoding transport systems and metabolic pathways (Figure 7), likely reflecting the carbon energy sources available in the intestine. Modulation of LPS, along with ShiA and OspI may enable Shigella to subvert inflammatory responses prior to mucosal invasion. Our results highlight the central role of oxygen and FNR in these processes and how it governs bacterial interactions and entry into host cells $[7,68]$.

\section{Methods}

\section{Bacterial strains and culture conditions}

Bacterial strains and plasmids used in this study are shown in Additional file 1: Table S4. E. coli strains were grown in Luria-Bertani (LB; Invitrogen) broth or on LB agar plates while $S$. flexneri was propagated either in LB broth, tryptic soy broth (TCS; Sigma) or on TCS plates with Congo red $(0.01 \%$, Sigma). Experiments under anaerobiosis were performed in an anaerobic workstation (Whitley A35). When required, antibiotics were added at the following concentrations: chloramphenicol $20 \mu \mathrm{g} / \mathrm{ml}$, ampicillin $100 \mu \mathrm{g} / \mathrm{ml}$.

\section{Deletion of fnr gene and complementation experiments}

The $f n r$ deletion mutant was generated by allelic exchange using pKO3blue plasmid as previously described [105]. Oligonucleotide primers used in this study are listed in Additional file 1: Table S5. Complementation of $\Delta f n r$ mutant was performed with $\mathrm{pBM} 2$, a derivative of pBBR1MCS-4 plasmid that carries a copy of fnr gene under the control of its native promoter. The plasmid pBBR1MCS-4 was used as a control (See Additional file 1: Table S4). The absence of FNR in the $\Delta f n r$ mutant and its presence in the complemented strain was confirmed by western blot using polyclonal antibodies against FNR as previously described [7] (See Additional file 1: Figure S1). 


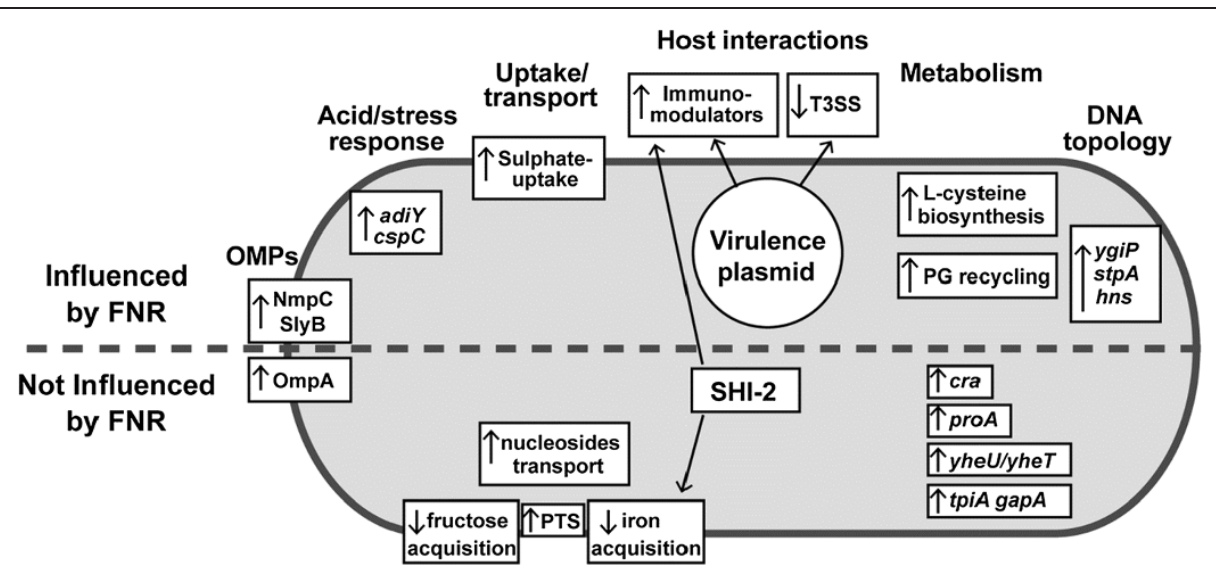

Figure 7 Summary of novel genes influenced by the absence of oxygen in Shigella identified by RNA-seq.

\section{DNA and RNA extraction methods}

S. flexneri M90T genomic DNA for sequencing was isolated as previously described [106]. For RNA extraction bacteria were grown in LB medium with and without oxygen. A $5 \mathrm{ml}$ pre-inoculum was grown over night aerobically or anaerobically with shaking conditions. The pre-inoculums were diluted proportionally to their $\mathrm{OD}_{600 \mathrm{~nm}}$ to standardize the input of bacteria to a starting $\mathrm{OD}_{600 \mathrm{~nm}}$ of 0.005 . Cultures (volume, $175 \mathrm{ml}$ in $1 \mathrm{~L}$ flasks) were grown at $37^{\circ} \mathrm{C}$, under shaking conditions (200 rpm) until the $\mathrm{OD}_{600 \mathrm{~nm}}$ reached 0.2 . Three biological replicates were performed for each condition. Total RNA from bacterial pellets was extracted using TRIzol reagent method as previously described [107]. RNA qualities were determined using Agilent RNA Nano Chips (Agilent Technologies).

Genomic DNA was removed from RNA samples using TURBO DNase (Ambion) followed by a second DNase treatment with DNase I (Roche). DNase I treatment was repeated until DNA was not detected by genomespecific PCRs targeting four housekeeping genes $(\operatorname{trp} B$, thrB, purN and $m d h$ ) (Additional file 1: Table S5). The RNA quality after DNase treatments was checked using Agilent RNA Nano Chips.

For RNA-seq, total RNA was reverse transcribed using SuperScript III reverse transcriptase (Invitrogen). Actinomycin D (6 $\mu \mathrm{g} / \mathrm{ml}$, Sigma) was added to the reaction to avoid spurious second-strand cDNA synthesis [108]. cDNA was purified using QIAquick PCR purification kit (Qiagen) and used for single stranded cDNA library construction as previously $[109,110]$. FRT-seq Illumina libraries were constructed as previously described [111].

\section{Reference genome, sequencing, read mapping and} statistic analysis

The genome of S. flexneri M90T was sequenced at Wellcome Trust Sanger Institute using an Illumina HiSeq 2000 sequencer. A total of $0.7 \mathrm{~Gb}$ sequence data, in 75-bp paired reads, was obtained (acc. no. ERS033387) and assembled de novo using Velvet [112]. This assembled sequence, which is rich in IS1 elements and for which no attempt of gap closure was performed, is comprised of 501 contigs with a total size of $4.43 \mathrm{Mb}$. A M90T draft annotated genome was prepared and the annotation transferred from S. flexneri strain 8401 (acc. no. CP000266). Rfam searches were performed and the features identified were included in the annotation as well as Shigella published sRNAs [69,70]. This draft genome was used as reference for the mapping of RNA-seq reads [113]. During the course of our study the S. flexneri M90T genome was published [114]. Therefore, final expression results are given using this latter locus tag systematic names for coding sequences.

RNA Sequencing was performed using an Illumina HiSeq 2000 sequencer. Raw data as well as mapped reads obtained per replicate were averaged per sample/ condition and summarized, together with other interesting quality control parameters, in Additional file 1: Table S3. Processing of reads after mapping included the unmarking of duplicate reads followed by correction to allow for directional fidelity of the data [115]. Output files included per sample, a matrix of readcounts and RPKM values on both sense and antisense strands for genes as well as for automatic $50 \mathrm{bp}+/-$ trimmed intergenic features created in the + strand. The $\mathrm{R}$ package DESeq, which implements negative binomial distribution statistics for RNA-seq data was used for statistical analysis [116]. A logarithmic transformed version of the count data $(\log (x+1))$ was used to avoid zero count values [117]. A $p$ adjust value $<0.05$, which controls false discovery rate, was used for the cut-off calling of differential expression between conditions. Independent runs of analysis were carried out for sense and antisense directions. Ribosomal genes and repeated sequences, such as transposases or insertion sequences, were filtered out from final tables. 


\section{Strand-specific quantitative RT-PCR and Northern blot} A StepOnePlus Real Time PCR system (Applied Biosystems) was used to monitor real-time quantitative PCR. First-strand cDNA was synthesized as previously described but using genome specific primers carrying a tag sequence in the $5^{\prime}$-end instead of random primers. This tag sequence was unique and not found in the genome of S. flexneri M90T. Subsequent PCRs were performed using Power SYBR Green PCR Master Mix (Applied Biosystems) and the tag sequence as one of the paired primers (See Additional file 1: Table S5). As a result, only cDNAs synthesized with a $5^{\prime}$-end tagged primer were amplified. Results are the average of triplicate experiments performed, on at least four independent occasions. Data were expressed relative to polA mRNA levels. To monitor the specificity, final PCR products were analyzed by melting curves. Only samples with no amplification in the control aliquots (not subjected to reverse transcription) were included in the study. The amount of transcripts was expressed as the $n$-fold difference relative to the control gene $\left(2^{-\Delta C t}\right.$ where $\Delta C t$ represents the difference in threshold cycles between the target and control genes). Results were shown in relation to wild type $2^{-\Delta \mathrm{Ct}}$ levels under aerobic conditions, which were referred as 1 . Thus, values greater than 1 indicate increased transcription in relation to the wild-type under aerobic conditions, and lower than 1 indicate the opposite. Significant differences were detected with Mann-Whitney test; values with $P<0.05$ were considered as significant.

Northern blots were performed as previously described [118]. Radiolabeled RNA probes synthesized with the MAXIscript kit (Ambion) were used to detect specifically the sense of the RNA-targets. The primers used for probes synthesis are listed in Additional file 1: Table S5.

\section{Availability of supporting data}

RNA-seq data has been submitted to the European Nucleotide Archive with accession code ERP003817 and the experiment has an ArrayExpress acc. no. E-ERAD-204.

\section{Additional file}

Additional file 1: Table S1. Chromosomal genes differentially expressed in response to anaerobic conditions and the role of FNR in the induction. This table shows the chromosomal genes differentially expressed in RNA-seq analysis in wild-type S. flexneri M90T grown under anaerobic conditions compared to aerobic conditions, and in $\Delta f h r$ mutant in relation to wild-type S. flexneri M90T when grown under anaerobic conditions. Genes are classified into functional categories based on the database of Clusters of Orthologous Groups (COGs). Table S2. FNR regulon under anaerobic conditions. This table contains all genes differentially expressed in the $\Delta \mathrm{fnr}$ mutant in relation to the wild-type S. flexneri M90T when grown under anaerobic conditions. RNA-seq and FRT-seq results are presented. Table S3. Summary of mapping statistics. Table S4. Strains and plasmids used in this study. Table S5. Oligonucleotides used in this study. Figure S1.

Characterization of M90T $\Delta f n r$ mutant. This figure confirms the absence of FNR in the $\Delta$ fnr mutant and shows the growth curve of the mutant in comparison to the wild-type strain M90T and the complemented mutant under anaerobic conditions.

Competing interests

The authors declare that they have no competing interests.

\section{Authors' contribution}

MV-I participated in the design of the study, performed the research, participated in the analysis of RNA-seq and FRT-seq data and drafted the manuscript. MCF prepared the genome assembly, performed the analysis of RNA-seq and FRT-seq data and helped on figures preparation. NRT participated in the design of the study and the analysis of RNA-seq and FRT-seq data. CMT conceived of the study and participated in its design and coordination and helped to draft the manuscript. All authors read and approved the final manuscript.

\section{Acknowledgements}

We thank Jeffrey A. Cole for his advice and suggestions, Iñigo Lasa for pKO3blue plasmid, David Harris and Nathalie Smerdon for data and ArrayExpress submissions; Lira Mamanova for the FRT-seq Illumina library construction; Lesley A. H. Bowman, Malene Cohen, Haifang Zhang and Nuria Vergara for their thoughtful reading of the manuscript and members of Tang group for their help.

M. Vergara-Irigaray was funded by FP7 Marie Curie EIMID-IAPP-217768 grant, and Stopenterics EU grant no. 261472. M. Fookes and N. Thomson are supported by Wellcome Trust grant 098051.

\section{Author details}

${ }^{1}$ Sir William Dunn School of Pathology, Oxford University, Oxford, United Kingdom. ${ }^{2}$ Centre for Molecular Microbiology and Infection, Imperial College London, London, United Kingdom. ${ }^{3}$ The Wellcome Trust Sanger Institute, Hinxton, Cambridgeshire, United Kingdom.

Received: 19 September 2013 Accepted: 23 May 2014

Published: 6 June 2014

\section{References}

1. Sansonetti PJ, Kopecko DJ, Formal SB: Involvement of a plasmid in the invasive ability of Shigella flexneri. Infect Immun 1982, 35(3):852-860.

2. Sansonetti PJ, Hale TL, Dammin GJ, Kapfer C, Collins HH Jr, Formal SB: Alterations in the pathogenicity of Escherichia coli K-12 after transfer of plasmid and chromosomal genes from Shigella flexneri. Infect Immun 1983, 39(3):1392-1402

3. Allen CA, Torres AG: Host-microbe communication within the GI tract. Adv Exp Med Biol 2008, 635:93-101.

4. Smith $\mathrm{H}$ : What happens to bacterial pathogens in vivo? Trends Microbiol 1998, 6(6):239-243.

5. Pederson KJ, Pierson DE: Ail expression in Yersinia enterocolitica is affected by oxygen tension. Infect Immun 1995, 63(10):4199-4201.

6. Boulette ML, Payne SM: Anaerobic regulation of Shigella flexneri virulence: ArcA regulates Fur and iron acquisition genes. J Bacterio/ 2007, 189(19):6957-6967.

7. Marteyn B, West NP, Browning DF, Cole JA, Shaw JG, Palm F, Mounier J, Prevost MC, Sansonetti P, Tang CM: Modulation of Shigella virulence in response to available oxygen in vivo. Nature 2010, 465(7296):355-358.

8. James BW, Keevil CW: Influence of oxygen availability on physiology, verocytotoxin expression and adherence of Escherichia coli 0157. J Appl Microbiol 1999, 86(1):117-124.

9. Diard S, Toribio AL, Boum Y, Vigier F, Kansau I, Bouvet O, Servin A: Environmental signals implicated in Dr fimbriae release by pathogenic Escherichia coli. Microbes Infect 2006, 8(7):1851-1858.

10. Singh RD, Khullar M, Ganguly NK: Role of anaerobiosis in virulence of Salmonella typhimurium. Mol Cell Biochem 2000, 215(1-2):39-46.

11. Khullar M, Singh RD, Smriti M, Ganguly NK: Anaerobiosis-induced virulence of Salmonella enterica subsp. enterica serovar Typhimurium: role of phospholipase Cgamma signalling cascade. J Med Microbiol 2003, 52(Pt 9):741-745.

12. Contreras I, Toro CS, Troncoso G, Mora GC: Salmonella typhi mutants defective in anaerobic respiration are impaired in their ability to replicate within epithelial cells. Microbiology 1997, 143(Pt 8):2665-2672. 
13. Krishnan HH, Ghosh A, Paul K, Chowdhury R: Effect of anaerobiosis on expression of virulence factors in Vibrio cholerae. Infect Immun 2004, 72(7):3961-3967

14. Kiley PJ, Beinert $\mathrm{H}$ : The role of Fe-S proteins in sensing and regulation in bacteria. Curr Opin Microbiol 2003, 6(2):181-185.

15. Whitehead RN, Overton TW, Snyder LA, McGowan SJ, Smith H, Cole JA, Saunders NJ: The small FNR regulon of Neisseria gonorrhoeae: comparison with the larger Escherichia coli FNR regulon and interaction with the NarQ-NarP regulon. BMC Genomics 2007, 8:35.

16. Constantinidou C, Hobman JL, Griffiths L, Patel MD, Penn CW, Cole JA Overton TW: A reassessment of the FNR regulon and transcriptomic analysis of the effects of nitrate, nitrite, NarXL, and NarQP as Escherichia coli K12 adapts from aerobic to anaerobic growth. J Biol Chem 2006, 281(8):4802-4815

17. Kang Y, Weber KD, Qiu Y, Kiley PJ, Blattner FR: Genome-wide expression analysis indicates that FNR of Escherichia coli K-12 regulates a large number of genes of unknown function. J Bacteriol 2005, 187(3):1135-1160.

18. Salmon K, Hung SP, Mekjian K, Baldi P, Hatfield GW, Gunsalus RP: Global gene expression profiling in Escherichia coli K12. The effects of oxygen availability and FNR. J Biol Chem 2003, 278(32):29837-29855.

19. Fink RC, Evans MR, Porwollik S, Vazquez-Torres A, Jones-Carson J, Troxell B, Libby SJ, McClelland M, Hassan HM: FNR is a global regulator of virulence and anaerobic metabolism in Salmonella enterica serovar Typhimurium (ATCC 14028 s). J Bacteriol 2007, 189(6):2262-2273.

20. Myers KS, Yan H, Ong IM, Chung D, Liang K, Tran F, Keles S, Landick R, Kiley P. Genome-scale analysis of Escherichia coli FNR reveals complex features of transcription factor binding. PLOS Genet 2013, 9(6):e1003565.

21. Rolfe MD, Rice CJ, Lucchini S, Pin C, Thompson A, Cameron AD, Alston M, Stringer MF, Betts RP, Baranyi J, Peck MW, Hinton JC: Lag phase is a distinct growth phase that prepares bacteria for exponential growth and involves transient metal accumulation. J Bacteriol 2012, 194(3):686-701.

22. Sezonov G, Joseleau-Petit D, D'Ari R: Escherichia coli physiology in Luria-Bertani broth. J Bacterio/ 2007, 189(23):8746-8749.

23. Mamanova L, Andrews RM, James KD, Sheridan EM, Ellis PD, Langford CF, Ost TW, Collins JE, Turner DJ: FRT-seq: amplification-free, strand-specific transcriptome sequencing. Nat Methods 2010, 7(2):130-132.

24. Tatusov RL, Galperin MY, Natale DA, Koonin EV: The COG database: a tool for genome-scale analysis of protein functions and evolution. Nucleic Acids Res 2000, 28(1):33-36.

25. Salmon KA, Hung SP, Steffen NR, Krupp R, Baldi P, Hatfield GW, Gunsalus RP: Global gene expression profiling in Escherichia coli K12: effects of oxygen availability and ArCA. J Biol Chem 2005, 280(15):15084-15096.

26. Gong S, Richard H, Foster JW: YjdE (AdiC) is the arginine:agmatine antiporter essential for arginine-dependent acid resistance in Escherichia coli. J Bacteriol 2003, 185(15):4402-4409.

27. Schweizer $\mathrm{H}$, Larson TJ: Cloning and characterization of the aerobic sn-glycerol-3-phosphate dehydrogenase structural gene glpD of Escherichia coli K-12. J Bacteriol 1987, 169(2):507-513.

28. Eshoo MW: lac fusion analysis of the bet genes of Escherichia coli: regulation by osmolarity, temperature, oxygen, choline, and glycine betaine. J Bacteriol 1988, 170(11):5208-5215.

29. Tchieu JH, Norris V, Edwards JS, Saier MH Jr: The complete phosphotransferase system in Escherichia coli. J Mol Microbiol Biotechnol 2001, 3(3):329-346.

30. Dahl U, Jaeger T, Nguyen BT, Sattler JM, Mayer C: Identification of a phosphotransferase system of Escherichia coli required for growth on N-acetylmuramic acid. J Bacteriol 2004, 186(8):2385-2392.

31. Hadi T, Dahl U, Mayer C, Tanner ME: Mechanistic studies on $\mathbf{N}$ acetylmuramic acid 6-phosphate hydrolase (MurQ): an etherase involved in peptidoglycan recycling. Biochemistry 2008, 47(44):11547-11558.

32. Jaeger T, Arsic M, Mayer C: Scission of the lactyl ether bond of $\mathrm{N}$-acetylmuramic acid by Escherichia coli "etherase". J Biol Chem 2005, 280(34):30100-30106.

33. Nishino K, Yamaguchi A: Analysis of a complete library of putative drug transporter genes in Escherichia coli. J Bacteriol 2001, 183(20):5803-5812.

34. Sirko A, Zatyka M, Sadowy E, Hulanicka D: Sulfate and thiosulfate transport in Escherichia coli K-12: evidence for a functional overlapping of sulfateand thiosulfate-binding proteins. J Bacterio/ 1995, 177(14):4134-4136.

35. Verkhovskaya ML, Barquera B, Wikstrom M: Deletion of one of two Escherichia coli genes encoding putative $\mathrm{Na}+/ \mathrm{H}+$ exchangers $(y c g O)$ perturbs cytoplasmic alkali cation balance at low osmolarity. Microbiology 2001, 147(Pt 11):3005-3013.

36. Casagrande F, Harder D, Schenk A, Meury M, Ucurum Z, Engel A, Weitz D, Daniel $H$, Fotiadis D: Projection structure of DtpD $(\mathrm{YbgH})$, a prokaryotic member of the peptide transporter family. J Mol Bio/ 2009, 394(4):708-717.

37. Hantke K: Phage T6-colicin K receptor and nucleoside transport in Escherichia coli. FEBS Lett 1976, 70(1):109-112.

38. Westh Hansen SE, Jensen N, Munch-Petersen A: Studies on the sequence and structure of the Escherichia coli K-12 nupG gene, encoding a nucleoside-transport system. Eur J Biochem 1987, 168(2):385-391.

39. Craig JE, Zhang Y, Gallagher MP: Cloning of the nupC gene of Escherichia coli encoding a nucleoside transport system, and identification of an adjacent insertion element, IS 186. Mol Microbiol 1994, 11(6):1159-1168.

40. Pritchard RH, Ahmad SI: Fluorouracil and the isolation of mutants lacking uridine phosphorylase in Escherichia coli: location of the gene. Mol Gen Genet 1971, 111(1):84-88.

41. Saier MH Jr, Ramseier TM: The catabolite repressor/activator (Cra) protein of enteric bacteria. J Bacterio/ 1996, 178(12):3411-3417.

42. Anderson A, Cooper RA: Gluconeogenesis in Escherichia coli The role of triose phosphate isomerase. FEBS Lett 1969, 4(1):19-20.

43. Branlant G, Branlant C: Nucleotide sequence of the Escherichia coli gap gene. Different evolutionary behavior of the NAD + -binding domain and of the catalytic domain of D-glyceraldehyde-3-phosphate dehydrogenase. Eur J Biochem 1985, 150(1):61-66.

44. Kraxenberger $\mathrm{T}$, Fried $\mathrm{L}$, Behr $\mathrm{S}$, Jung K: First insights into the unexplored two-component system YehU/YehT in Escherichia coli. J Bacteriol 2012, 194(16):4272-4284

45. Boos W, Shuman H: Maltose/maltodextrin system of Escherichia coli: transport, metabolism, and regulation. Microbiol Mol Biol Rev 1998 62(1):204-229.

46. Baich A: Proline synthesis in Escherichia coli.A proline-inhibitable glutamic acid kinase. Biochim Biophys Acta 1969, 192(3):462-467.

47. Baich A: The biosynthesis of proline in Escherichia coli: phosphatedependent glutamate -semialdehyde dehydrogenase (NADP), the second enzyme in the pathway. Biochim Biophys Acta 1971, 244(1):129-134.

48. Atlung $\mathrm{T}$, Ingmer $\mathrm{H}$ : H-NS: a modulator of environmentally regulated gene expression. Mol Microbio/ 1997, 24(1):7-17.

49. Cohen-Or I, Shenhar Y, Biran D, Ron EZ: CspC regulates rpoS transcript levels and complements hfq deletions. Res Microbiol 2010, 161(8):694-700.

50. Molloy MP, Herbert BR, Walsh BJ, Tyler MI, Traini M, Sanchez JC, Hochstrasser DF, Williams KL, Gooley AA: Extraction of membrane proteins by differential solubilization for separation using two-dimensional gel electrophoresis. Electrophoresis 1998, 19(5):837-844.

51. Pugsley AP, Schnaitman CA: Identification of three genes controlling production of new outer membrane pore proteins in Escherichia coli K-12. J Bacterio/ 1978, 135(3):1118-1129.

52. Chai TJ, Foulds J: Purification of protein A, an outer membrane component missing in Escherichia coli K-12 ompA mutants. Biochim Biophys Acta 1977, 493(1):210-215.

53. Ludwig A, Tengel C, Bauer S, Bubert A, Benz R, Mollenkopf HJ, Goebel W: SlyA, a regulatory protein from Salmonella typhimurium, induces a haemolytic and pore-forming protein in Escherichia coli. Mol Gen Genet 1995, 249(5):474-486.

54. Gao J, Lee K, Zhao M, Qiu J, Zhan X, Saxena A, Moore CJ, Cohen SN, Georgiou G: Differential modulation of E. coli mRNA abundance by inhibitory proteins that alter the composition of the degradosome. Mol Microbiol 2006, 61(2):394-406.

55. Polikanov YS, Blaha GM, Steitz TA: How hibernation factors RMF, HPF, and YfiA turn off protein synthesis. Science 2012, 336(6083):915-918.

56. Lee C, Marians KJ: Characterization of the nucleoid-associated protein YejK. J Biol Chem 2013, 288(44):31503-31516.

57. Teramoto J, Yoshimura SH, Takeyasu K, Ishihama A: A novel nucleoid protein of Escherichia coli induced under anaerobiotic growth conditions. Nucleic Acids Res 2010, 38(11):3605-3618.

58. Unden G: Differential roles for menaquinone and demethylmenaquinone in anaerobic electron transport of $E$. coli and their fnr-independent expression. Arch Microbiol 1988, 150(5):499-503.

59. Shestopalov Al, Bogachev AV, Murtazina RA, Viryasov MB, Skulachev VP: Aeration-dependent changes in composition of the quinone pool in Escherichia coli. Evidence of post-transcriptional regulation of the quinone biosynthesis. FEBS Lett 1997, 404(2-3):272-274. 
60. Sharma V, Hudspeth ME, Meganathan R: Menaquinone (vitamin K2) biosynthesis: localization and characterization of the menE gene from Escherichia coli. Gene 1996, 168(1):43-48.

61. Gruez A, Zeghouf M, Bertrand J, Eschenbrenner M, Coves J, Fontecave M Pignol D, Fontecilla-Camps JC: The FNR-like domain of the Escherichia coli sulfite reductase flavoprotein component: crystallization and preliminary X-ray analysis. Acta Crystallogr D Biol Crystallogr 1998, 54(Pt 1):135-136.

62. Sirko A, Hryniewicz M, Hulanicka D, Bock A: Sulfate and thiosulfate transport in Escherichia coli K-12: nucleotide sequence and expression of the cysTWAM gene cluster. J Bacterio/ 1990, 172(6):3351-3357.

63. Leyh TS, Taylor JC, Markham GD: The sulfate activation locus of Escherichia coli K12: cloning, genetic, and enzymatic characterization. J Biol Chem 1988, 263(5):2409-2416.

64. Loughlin RE: Polarity of the cys JH operon of Salmonella typhimurium. J Gen Microbiol 1975, 86(2):275-282.

65. Abeles AL, Friedman SA, Austin SJ: Partition of unit-copy miniplasmids to daughter cells. III. The DNA sequence and functional organization of the P1 partition region. J Mol Biol 1985, 185(2):261-272.

66. Moss JE, Cardozo TJ, Zychlinsky A, Groisman EA: The selC-associated SHI-2 pathogenicity island of Shigella flexneri. Mol Microbiol 1999, 33(1):74-83.

67. Lemaitre $C$, Bidet $P$, Bingen $E$, Bonacorsi S: Transcriptional analysis of the Escherichia coli ColV-la plasmid pS88 during growth in human serum and urine. BMC Microbiol 2012, 12:115.

68. Ingersoll MA, Moss JE, Weinrauch Y, Fisher PE, Groisman EA, Zychlinsky A The ShiA protein encoded by the Shigella flexneri SHI-2 pathogenicity island attenuates inflammation. Cell Microbiol 2003, 5(11):797-807.

69. Peng J, Yang J, Jin Q: An integrated approach for finding overlooked genes in Shigella. PLoS One 2011, 6(4):e18509.

70. Skippington E, Ragan MA: Evolutionary dynamics of small RNAs in 27 Escherichia coli and Shigella genomes. Genome Biol Evol 2012, 4(3):330-345

71. Kroger C, Dillon SC, Cameron AD, Papenfort K, Sivasankaran SK, Hokamp K, Chao Y, Sittka A, Hebrard M, Handler K, Colgan A, Leekitcharoenphon P, Langridge GC, Lohan AJ, Loftus B, Lucchini S, Ussery DW, Dorman CJ, Thomson NR, Vogel J, Hinton JC: The transcriptional landscape and small RNAs of Salmonella enterica serovar Typhimurium. Proc Natl Acad Sci USA 2012, 109(20):E1277-E1286.

72. Gore AL, Payne SM: CsrA and Cra influence Shigella flexneri pathogenesis. Infect Immun 2010, 78(11):4674-4682.

73. Njoroge JW, Nguyen Y, Curtis MM, Moreira CG, Sperandio V: Virulence meets metabolism: Cra and $\mathrm{KdpE}$ gene regulation in enterohemorrhagic Escherichia coli. MBio 2012, 3(5):e00280-12.

74. Allen JH, Utley M, van Den Bosch H, Nuijten P, Witvliet M, McCormick BA, Krogfelt KA, Licht TR, Brown D, Mauel M, Leatham MP, Laux DC, Cohen PS: A functional cra gene is required for Salmonella enterica serovar typhimurium virulence in BALB/c mice. Infect Immun 2000, 68(6):3772-3775.

75. Eisenreich W, Dandekar T, Heesemann J, Goebel W: Carbon metabolism of intracellular bacterial pathogens and possible links to virulence. Nat Rev Microbiol 2010, 8(6):401-412.

76. Joseph B, Przybilla K, Stuhler C, Schauer K, Slaghuis J, Fuchs TM, Goebel W: Identification of Listeria monocytogenes genes contributing to intracellular replication by expression profiling and mutant screening. J Bacterio/ 2006, 188(2):556-568.

77. Marrero J, Rhee KY, Schnappinger D, Pethe K, Ehrt S: Gluconeogenic carbon flow of tricarboxylic acid cycle intermediates is critical for Mycobacterium tuberculosis to establish and maintain infection. Proc Natl Acad Sci USA 2010, 107(21):9819-9824.

78. Tang Y, Wu W, Zhang X, Lu Z, Chen J, Fang W: Catabolite control protein a of Streptococcus suis type 2 contributes to sugar metabolism and virulence. J Microbiol 2012, 50(6):994-1002.

79. Heroven AK, Sest M, Pisano F, Scheb-Wetzel M, Steinmann R, Bohme K, Klein J, Munch R, Schomburg D, Dersch P: Crp Induces Switching of the CsrB and CsrC RNAs in Yersinia pseudotuberculosis and Links Nutritional Status to Virulence. Front Cell Infect Microbio/ 2012, 2:158.

80. Altier C, Suyemoto M, Lawhon SD: Regulation of Salmonella enterica serovar typhimurium invasion genes by csrA. Infect Immun 2000, 68(12):6790-6797.

81. Gotz A, Goebel W: Glucose and glucose 6-phosphate as carbon sources in extra- and intracellular growth of enteroinvasive Escherichia coli and Salmonella enterica. Microbiology 2010, 156(Pt 4):1176-1187.
82. Kieboom J, Abee T: Arginine-dependent acid resistance in Salmonella enterica serovar Typhimurium. J Bacteriol 2006, 188(15):5650-5653.

83. Lin J, Lee IS, Frey J, Slonczewski JL, Foster JW: Comparative analysis of extreme acid survival in Salmonella typhimurium, Shigella flexneri, and Escherichia coli. J Bacteriol 1995, 177(14):4097-4104.

84. Casalino M, Prosseda G, Barbagallo M, lacobino A, Ceccarini P, Latella MC, Nicoletti M, Colonna B: Interference of the CadC regulator in the argininedependent acid resistance system of Shigella and enteroinvasive E. coli. Int J Med Microbiol 2010, 300(5):289-295.

85. Maurelli AT, Fernandez RE, Bloch CA, Rode CK, Fasano A: "Black holes" and bacterial pathogenicity: a large genomic deletion that enhances the virulence of Shigella spp. and enteroinvasive Escherichia coli. Proc Natl Acad Sci USA 1998, 95(7):3943-3948.

86. Grainger DC, Aiba H, Hurd D, Browning DF, Busby SJ: Transcription factor distribution in Escherichia coli: studies with FNR protein. Nucleic Acids Res 2007, 35(1):269-278.

87. Waters LS, Storz G: Regulatory RNAs in bacteria. Cell 2009, 136(4):615-628.

88. Weilbacher T, Suzuki K, Dubey AK, Wang X, Gudapaty S, Morozov I, Baker CS, Georgellis D, Babitzke P, Romeo T: A novel sRNA component of the carbon storage regulatory system of Escherichia coli. Mol Microbiol 2003, 48(3):657-670.

89. Liu MY, Gui G, Wei B, Preston JF 3rd, Oakford L, Yuksel U, Giedroc DP, Romeo T: The RNA molecule CsrB binds to the global regulatory protein CsrA and antagonizes its activity in Escherichia coli. J Biol Chem 1997, 272(28):17502-17510.

90. Tobe T, Yoshikawa M, Mizuno T, Sasakawa C: Transcriptional control of the invasion regulatory gene virB of Shigella flexneri: activation by virF and repression by H-NS. J Bacteriol 1993, 175(19):6142-6149.

91. Perdomo OJ, Cavaillon JM, Huerre M, Ohayon H, Gounon P, Sansonetti PJ: Acute inflammation causes epithelial invasion and mucosal destruction in experimental shigellosis. J Exp Med 1994, 180(4):1307-1319.

92. Perdomo JJ, Gounon P, Sansonetti PJ: Polymorphonuclear leukocyte transmigration promotes invasion of colonic epithelial monolayer by Shigella flexneri. J Clin Invest 1994, 93(2):633-643.

93. Ingersoll MA, Zychlinsky A: ShiA abrogates the innate T-cell response to Shigella flexneri infection. Infect Immun 2006, 74(4):2317-2327.

94. Sanada T, Kim M, Mimuro H, Suzuki M, Ogawa M, Oyama A, Ashida H, Kobayashi T, Koyama T, Nagai S, Shibata Y, Gohda J, Inoue J, Mizushima T, Sasakawa C: The Shigella flexneri effector Ospl deamidates UBC13 to dampen the inflammatory response. Nature 2012, 483(7391):623-626.

95. Kaoukab-Raji A, Biskri L, Bernardini ML, Allaoui A: Characterization of SfPgdA, a Shigella flexneri peptidoglycan deacetylase required for bacterial persistence within polymorphonuclear neutrophils. Microbes Infect 2012, 14(7-8):619-627.

96. Kaniuk NA, Vinogradov E, Li J, Monteiro MA, Whitfield C: Chromosomal and plasmid-encoded enzymes are required for assembly of the R3-type core oligosaccharide in the lipopolysaccharide of Escherichia coli 0157:H7. J Biol Chem 2004, 279(30):31237-31250.

97. Wing HJ, Goldman SR, Ally S, Goldberg MB: Modulation of an outer membrane protease contributes to the virulence defect of Shigella flexneri strains carrying a mutation in the virk locus. Infect Immun 2005, 73(2):1217-1220

98. D'Hauteville H, Khan S, Maskell DJ, Kussak A, Weintraub A, Mathison J, Ulevitch RJ, Wuscher N, Parsot C, Sansonetti PJ: Two msbB genes encoding maximal acylation of lipid A are required for invasive Shigella flexneri to mediate inflammatory rupture and destruction of the intestinal epithelium. J Immunol 2002, 168(10):5240-5251.

99. Zhong QP: Pathogenic effects of Opolysaccharide from Shigella flexneri strain. World J Gastroenterol 1999, 5(3):245-248.

100. Bernardini ML, Sanna MG, Fontaine A, Sansonetti PJ: OmpC is involved in invasion of epithelial cells by Shigella flexneri. Infect Immun 1993, 61(9):3625-3635.

101. Ambrosi C, Pompili M, Scribano D, Zagaglia C, Ripa S, Nicoletti M: Outer membrane protein $\mathrm{A}(\mathrm{OmpA})$ : a new player in shigella flexneri protrusion formation and inter-cellular spreading. PLoS One 2012, 7(11):e49625.

102. Singh R, Shasany AK, Aggarwal A, Sinha S, Sisodia BS, Khanuja SP, Misra R: Low molecular weight proteins of outer membrane of Salmonella typhimurium are immunogenic in Salmonella induced reactive arthritis revealed by proteomics. Clin Exp Immunol 2007, 148(3):486-493.

103. Yang TC, Ma XC, Liu F, Lin LR, Liu LL, Liu GL, Tong ML, Fu ZG, Zhou L: Screening of the Salmonella paratyphi A CMCC 50973 strain outer 
membrane proteins for the identification of potential vaccine targets. Mol Med Rep 2012, 5(1):78-83.

104. Egea L, Aguilera L, Gimenez R, Sorolla MA, Aguilar J, Badia J, Baldoma L: Role of secreted glyceraldehyde-3-phosphate dehydrogenase in the infection mechanism of enterohemorrhagic and enteropathogenic Escherichia coli: interaction of the extracellular enzyme with human plasminogen and fibrinogen. Int J Biochem Cell Biol 2007, 39(6):1190-1203.

105. Solano C, Garcia B, Latasa C, Toledo-Arana A, Zorraquino V, Valle J, Casals J, Pedroso E, Lasa I: Genetic reductionist approach for dissecting individual roles of GGDEF proteins within the c-di-GMP signaling network in Salmonella. Proc Natl Acad Sci USA 2009, 106(19):7997-8002.

106. Marmur J: A procedure for the isolation of Deoxyribonucleic acid from micro-organisms. J Mol Biol 1961, 3:208-218.

107. Toledo-Arana A, Dussurget O, Nikitas G, Sesto N, Guet-Revillet H, Balestrino D, Loh E, Gripenland J, Tiensuu T, Vaitkevicius K, Barthelemy M, Vergassola M, Nahori MA, Soubigou G, Regnault B, Coppee JY, Lecuit M, Johansson J, Cossart P: The Listeria transcriptional landscape from saprophytism to virulence. Nature 2009, 459(7249):950-956.

108. Perocchi F, Xu Z, Clauder-Munster S, Steinmetz LM: Antisense artifacts in transcriptome microarray experiments are resolved by actinomycin $\mathrm{D}$. Nucleic Acids Res 2007, 35(19):e128.

109. Croucher NJ, Fookes MC, Perkins TT, Turner DJ, Marguerat SB, Keane T, Quail MA, He M, Assefa S, Bahler J, Kingsley RA, Parkhill J, Bentley SD, Dougan G, Thomson NR: A simple method for directional transcriptome sequencing using Illumina technology. Nucleic Acids Res 2009, 37(22):e148.

110. Quail MA, Kozarewa I, Smith F, Scally A, Stephens PJ, Durbin R, Swerdlow H, Turner DJ: A large genome center's improvements to the lllumina sequencing system. Nat Methods 2008, 5(12):1005-1010.

111. Mamanova L, Turner DJ: Low-bias, strand-specific transcriptome Illumina sequencing by on-flowcell reverse transcription (FRT-seq). Nat Protoc 2011, 6(11):1736-1747.

112. Zerbino DR, Birney E: Velvet: algorithms for de novo short read assembly using de Bruijn graphs. Genome Res 2008, 18(5):821-829.

113. Li H, Handsaker B, Wysoker A, Fennell T, Ruan J, Homer N, Marth G, Abecasis G, Durbin R: The Sequence Alignment/Map format and SAMtools. Bioinformatics 2009, 25(16):2078-2079.

114. Onodera NT, Ryu J, Durbic T, Nislow C, Archibald JM, Rohde JR: Genome sequence of Shigella flexneri serotype 5a strain M90T Sm. J Bacteriol 2012, 194(11):3022.

115. Croucher NJ, Vernikos GS, Parkhill J, Bentley SD: Identification, variation and transcription of pneumococcal repeat sequences. BMC Genomics 2011, 12:120.

116. Anders $S$, Huber W: Differential expression analysis for sequence count data. Genome Biol 2010, 11(10):R106.

117. Simon Anders WH: Diferential expression of RNA-Seq data at the gene level -the DESeq package. 2013, URL: http://www.bioconductor.org/ packages/devel/bioc/vignettes/DESeq/inst/doc/DESeq.pdf.

118. Lasa I, Toledo-Arana A, Dobin A, Villanueva M, de los Mozos IR, Vergara-lrigaray M, Segura V, Fagegaltier D, Penades JR, Valle J, Solano C, Gingeras TR: Genome-wide antisense transcription drives mRNA processing in bacteria. Proc Natl Acad SCi USA 2011, 108(50):20172-20177.

doi:10.1186/1471-2164-15-438

Cite this article as: Vergara-lrigaray et al:: RNA-seq analysis of the influence of anaerobiosis and FNR on Shigella flexneri. BMC Genomics 2014 15:438

\section{Submit your next manuscript to BioMed Central and take full advantage of:}

- Convenient online submission

- Thorough peer review

- No space constraints or color figure charges

- Immediate publication on acceptance

- Inclusion in PubMed, CAS, Scopus and Google Scholar

- Research which is freely available for redistribution 